\title{
Comparison of gene expression in the gill of salmon (Salmo salar) smolts from anadromous and landlocked populations
}

\author{
Juha Lemmetyinen ${ }^{1}$, Jorma Piironen², Päivi Kiiskinen ${ }^{1}$, Minna Hassinen ${ }^{1}$ \& \\ Matti Vornanen ${ }^{1, *}$ \\ 1) University of Eastern Finland, Department of Biology, P.O. Box 111, Fl-80101 Joensuu, Finland \\ (*corresponding author's e-mail: matti.vornanen@uef.fi) \\ 2) Finnish Game and Fisheries Research Institute, Yliopistokatu 6, Fl-80100 Joensuu, Finland
}

Received 17 Feb. 2012, final version received 31 July 2012, accepted 21 Aug. 2012

Lemmetyinen, J., Piironen, J., Kiiskinen, P., Hassinen, M. \& Vornanen, M. 2013: Comparison of gene expression in the gill of salmon (Salmo salar) smolts from anadromous and landlocked populations. - Ann. Zool. Fennici 50: 16-35.

We examined whether gene expression in the young salmon (Salmo salar) gill differs in relation to the salinity of their migration habitat by comparing three salmon stocks: (1) fish that migrate from a river system to Lake Saimaa, (2) fish that migrate to the brackish waters of the Baltic Sea, and (3) fish that migrate to the full-strength salinity of the Arctic Ocean. Transcripts of the gill tissue were measured at three successive developmental stages (parr, smolt and postsmolt) using the cDNA microarray in fish reared under common conditions. The changes in gene expression were qualitatively and quantitatively similar in the three stocks irrespective of the salinity of the natural growing habitat. This suggests that the parr-smolt transformation in the gill tissue of the landlocked fresh-water salmon stock is similar to the seawater migrating salmon. The transformation of the gill to a hypoosmotic organ in the freshwater salmon has been retained in evolution, possibly due to its adaptive role as a signal for migration from a relatively poor-growth environment of the river to a more productive lake habitat.

\section{Introduction}

Anadromous salmonid fish migrate from freshwater rivers to seawater for rapid growth in the nutrient rich ocean habitat and return a few years later to the same stream for reproduction (Thorpe 1994). Migration of young salmon to sea is associated with major changes in morphology, physiology and behaviour of the fish, collectively called the parr-smolt transformation (smoltification), which remodels the young freshwater-adapted fish (parr) to the seawateradapted fish (smolt) (Hoar 1976, Folmar \& Dickhoff 1980, McCormick \& Saunders 1987). The parr-smolt transformation occurs in spring under the control of hormonal system entrained by increasing day-length and rising water temperature (Komourdian et al. 1976, Ban et al. 2007). Although smoltification happens only once in the lifetime of the wild fish, it is a reversible process that can occur several times under hatchery conditions. If access to seawater is pre- 
vented the smolt characteristics are disassembled and the smolt transforms to the postsmolt state (desmoltification) with many characteristics of the parr (Hoar 1976).

The parr-smolt transformation prepares the fish for migration from hypoosmotic freshwater to hyperosmotic seawater and is associated with a profound change in salt tolerance and ion regulation including a thorough transformation of the gill from an ion-absorbing to an ion-secreting organ (Parry 1960, Langdon 1985). Remodelling of the gill in the parr-smolt transformation is associated with large changes in expression and function of ion-transport molecules of the mitochondria-rich chloride cells and/or pavement cells including $\mathrm{Na}, \mathrm{K}$-ATPase, $\mathrm{Na}, \mathrm{K}, 2 \mathrm{Cl}$ transporter, cystic fibrosis transmembrane conductance regulator, V-type $\mathrm{H}$-ATPase and $\mathrm{Cl} /$ $\mathrm{HCO}_{3}$ exchanger (Saunders \& Henderson 1978, Boeuf et al. 1985, Sullivan et al. 1996, Seidelin et al. 2001, Evans et al. 2005, Nilsen et al. 2007, Madsen et al. 2009). This transformation of gill function represents a profound change in the physiology of the fish and is energetically costly (Hoar 1976, McCormick \& Saunders 1987). In addition to the seawater-migrating or anadromous populations of the Atlantic salmon, there are several landlocked salmon stocks which complete their whole life-cycle in the freshwater environment. The landlocked forms of the Atlantic salmon were derived from anadromous populations which lost their access to the sea due to the elevation of the land after the latest glaciation event some 5000-10 000 years ago (Berg 1985). Many of these populations spawn in upstream rivers and the juvenile salmon migrate to the downstream lake for growth, i.e. these salmon are still migratory but now migrate from one freshwater environment to another without any salinity change. Yet, the non-anadromous salmon populations go through a similar smoltification process as the anadromous salmon, although there seems to be differences between landlocked stocks in the extent of the parr-smolt transformation (Berg 1985, McDowall 1988, Birt et al. 1991, Staurnes et al. 1992, Birt \& Green 1993, Schmitz 1995, Kiiskinen et al. 2002). Indeed, recent findings suggest that in some landlocked salmon populations hormonal signals and ion regulatory changes in the smolts are weaker than in anadromous strains possibly as a consequence of negative selection pressure on the smolt traits (Nilsen et al. 2007, Nilsen et al. 2008).

Although several aspects of the parr-smolt transformation have been extensively examined, to our knowledge there are no high-throughput transcriptomic analyses of the parr-smolt transformation of landlocked and anadromous salmon populations (Vornanen et al. 2009, Seear et al. 2010). Therefore, the present study was designed to compare transcriptomes of the gill filaments of three different Atlantic salmon stocks that migrate from freshwater streams to a freshwater lake (Lake Saimaa, Finland), from the River Neva (Russia) to the brackish water of the Baltic Sea and from the Teno River (Finland/ Norway) to the full-strength seawater of the Arctic Ocean, respectively. The three strains of the same salmon species (Salmo salar) were reared together under standard salmon aquaculture conditions in freshwater. Gill samples were collected from parr, smolt and postsmolt fish for gene expression analysis on the salmonid GRASP 16K microarray platform (von Schalburg et al. 2005). Specifically, it was hypothesized that in salmon stocks that migrate to freshwater (the landlocked stock, LS) or brackish water (BS) the changes in transcript expression would be blunted in comparison to fish that migrate to the full-strength seawater (SS).

\section{Material and methods}

\section{Animal origin and care}

Three Atlantic salmon (Salmo salar) stocks that differed in regard to their geographical origin and growth habitat, but reared together under common conditions, were used for these experiments: the landlocked non-anadromous and freshwater-adapted Lake Saimaa salmon (landlocked stock, LS), and two anadromous sea-run populations: the Neva salmon (brackish-water stock, BS) and the Teno salmon (seawater stock, SS), adapted to brackish water and full-strength seawater, respectively. The origin and rearing conditions of the sampled salmon populations are described in detail in Piironen et al. (2013). All 


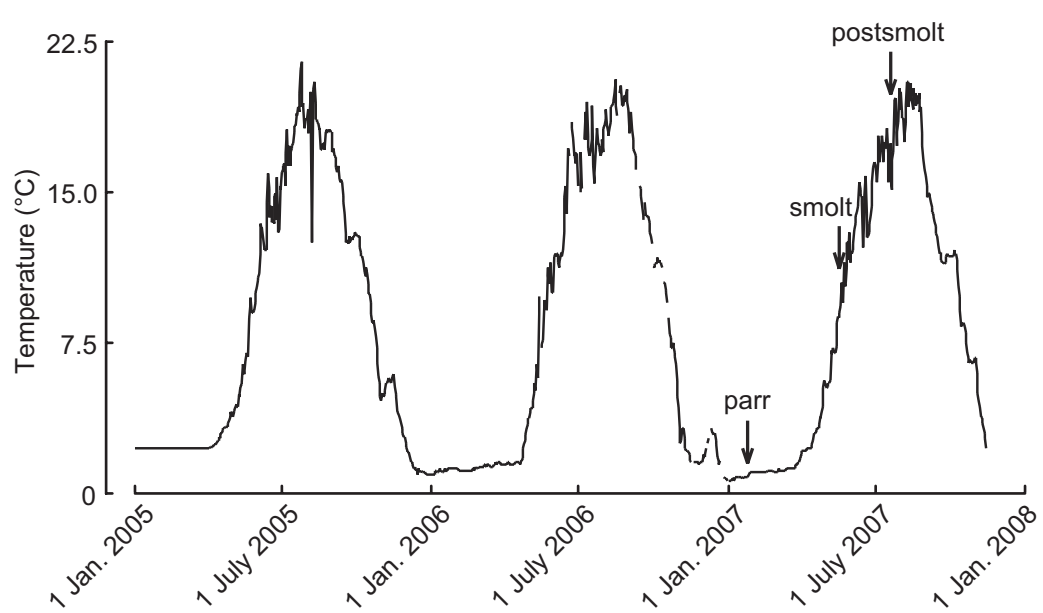

Fig. 1. Water temperature in the indoor tanks of the Enonkoski fish hatchery during the study giving the whole thermal history of the fish. The times of the gill-sample collection are indicated by arrows. experiments were conducted with permission of the ethical committee of the Game and Fisheries Research Institute (permission 18/05).

\section{Gill sampling and RNA isolation}

The fish were used for experiments at the age of $1+$ and represented the lower modal group (body mass 40.9-132.5 g). The gill tissue was collected at three different times during the development of the fish. The juvenile parr were sampled on 25 January, smolts on 15 May and the postsmolt fish on 17 July 2007. Water temperatures on the sampling days were $0.8,8.5$ and $15.5^{\circ} \mathrm{C}$, respectively (Fig. 1). Smolting of the fish was followed by measuring seasonal changes in the activity of gill $\mathrm{Na}, \mathrm{K}$-ATPase and the number of gill Napumps $\left(\left[{ }^{3} \mathrm{H}\right]\right.$ ouabain binding; for methods see Piironen et al. 2013) 5 times during the 1st year of development $(0+)$ and 4 times during the 2 nd year of development $(1+)$. In addition, developmental changes in $\mathrm{Na}$,K-ATPase activity and Napump density of individual fish were followed by taking gill biopsies ( 6 time points) from $8-10$ fish for each stock (results not shown). The smolt fish were sampled when gill Na,K-ATPase and Na-pump density reached their maximum values which occurred on the same date for all salmon stocks. External morphological characteristics of smoltification including condition factor and silvering of the skin were also recorded.

The fish were stunned by a blow to the head and killed by cervical dislocation. The third and fourth gill arches were excised from the left side of the gill and snapfrozen in liquid nitrogen for storage at $-80{ }^{\circ} \mathrm{C}$ until used for the RNA isolation. About $100 \mathrm{mg}$ of gill filaments were pooled from ten fishes for each stock, the tissue was homogenized in liquid nitrogen, divided into two equal aliquots, and the total RNA was extracted in two sets using Trizol reagent (Invitrogen, San Diego) according to the manufacturer's instructions. Integrity of the RNA was checked with gel electrophoresis, and quantity and purity of the RNA were analyzed using a NanoDrop ND-1000 UV/Vis spectrophotometer (Thermo Fisher Scientific, USA).

\section{Microarray}

Microarray experiments were conducted using the GRASP 16K cDNA array (von Schalburg et al. 2005). Detailed information on this chip can be found at http://web.uvic.ca/grasp/. Altogether 30 hybridizations were made on the nine RNA samples ( 3 salmon populations $\times 3$ time points), plus some extra hybridization to compensate for lower quality hybridizations (Fig. 2). All three populations at the same developmental stage (parr, smolt, postsmolt) were hybridized with each other, and within each population the three sequential developmental stages were hybridized with each other. All hybridizations were done using the pooled samples and in duplicates (dye swap).

RNA sample labeling and hybridization were performed as described by Brosché et al. (Bro- 
sché et al. 2005). RNA samples $(25 \mu \mathrm{g})$ were labeled by coupling of $\mathrm{Cy}^{\circledR}$ dyes (GE Healthcare, Buckinghamshire, UK) to the aminoallyldUTP-labeled cDNA, and subsequently the labeled cDNA was purified using the QIAGEN ${ }^{\circledR}$ QIAquick ${ }^{\circledR}$ PCR Purification Kit (Qiagen, Hilden, Germany). DNAs were hybridized in a mixture of $32.5 \mu \mathrm{l}$ formamide, $16.25 \mu 120 \times \mathrm{SSC}$, $3.25 \mu 12 \%$ SDS, $6.5 \mu 1$ herring sperm DNA (1 $\mathrm{mg} \mathrm{ml}^{-1}$ ) and $6.5 \mu 150 \times$ Denhardts. Slides were hybridized at $42{ }^{\circ} \mathrm{C}$ overnight (14-16 hours). After hybridization, the microarray slides were briefly dipped in $2 \times \mathrm{SSC} / 0.1 \%$ SDS until the LifterSlip fell off. The arrays were washed in $2 \times$ SSC, $0.1 \%$ SDS for $15 \mathrm{~min}, 1 \times$ SSC for 2 $\mathrm{min}, 0.2 \times \mathrm{SSC}$ for $1 \mathrm{~min}, 0.05 \times \mathrm{SSC}$ for $10 \mathrm{~s}$. Finally, the washed slides were dried by centrifuging for $5 \mathrm{~min}$ at $4000 \mathrm{rpm}$ in a slide centrifuge (Galaxy MiniArray, VWR, Pennsylvania, USA). All washing steps were performed at room temperature. The microarray experiment is compliant with the Minimum Information About a Microarray Experiment guidelines (MIAME) (Brazma et al. 2001). Complete protocols for probe labeling and hybridization, and the raw and combined data files are available from the ArrayExpress database (www.ebi.ac.uk/arrayexpress) under the accession number E-MEXP-2210.

Hybridized arrays were scanned with a ScanArray Gx scanner (PerkinElmer, Massachusetts, USA) at the $10-\mu \mathrm{m}$ resolution. The Cy3 and $\mathrm{Cy} 5$ cyanine fluorophores were excited at 543 and $633 \mathrm{~nm}$, respectively. The sensitivity (PMT and laser power) was adjusted individually to each array and both labels to obtain as much information as possible. The spot intensities were quantified with the ScanArray Express software (PerkinElmer, Massachusetts, USA), and median values of signal and background were used in the calculations. The statistical analysis and data normalization of the microarray were conducted according to "the direct two-color design" using R (2.10.1)/Bioconductor package LIMMA (2.12.0) (Linear Model for Microarray Data) (Smyth 2005). The background effect was subtracted using the "normexp" correction method (offset $=50$ ), and the data were normalized within the array with the "printTipLoess" method and between the arrays with the "quantile" method. Gene ontology (GO) anno-

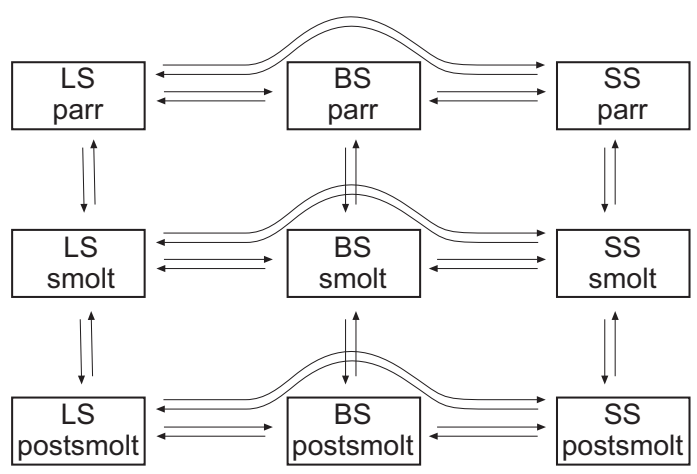

Fig. 2. A microarray hybridization design for comparison of salmon stocks at three developmental stages during the parr-smolt-postsmolt transformation. Each arrow indicates one hybridization. Arrows running in an opposite direction indicate the dye swap. LS = landlocked stock, BS = brackish-water stock, SS = seawater stock

tation was based on information available at http://web.uvic.ca/grasp/ or an analysis by the Blast2GO software (http://www.blast2go.org) (Götz et al. 2008).

\section{Validation of microarray data by qPCR}

A subset of 10 genes with significantly altered expression levels and different developmental patterns (CB489663, CB510827, CB501150, CA041067, CB506101, CB510517, CB500560, CB487042, CA048728, CB489182) were chosen for validation of the microarray data with quantitative real time PCR (qPCR) (Rockett and Hellmann 2004). DnaJA2 (DnaJ subfamily A member 2) was used as a reference gene. In studies of salmonid fish, DnaJA2 has turned out be more stable than e.g. the genes of $\beta$-actin or ribosomal RNA and similar to the elongation factor $1 \alpha$ during the parr-smolt transformation (Vornanen et al. 2005, Hassinen et al. 2007). The same RNA samples analyzed in the microarray were also used for qPCR, and represented the gill tissue pooled from 10 fishes (see Gill sampling and RNA isolation). For qPCR, the contaminating DNA was removed using RQ1 RNase-Free DNase (Promega, Madison, WI), and the absence of contamination was verified for each sample in a control run containing all other reaction components except the reverse transcriptase. The 

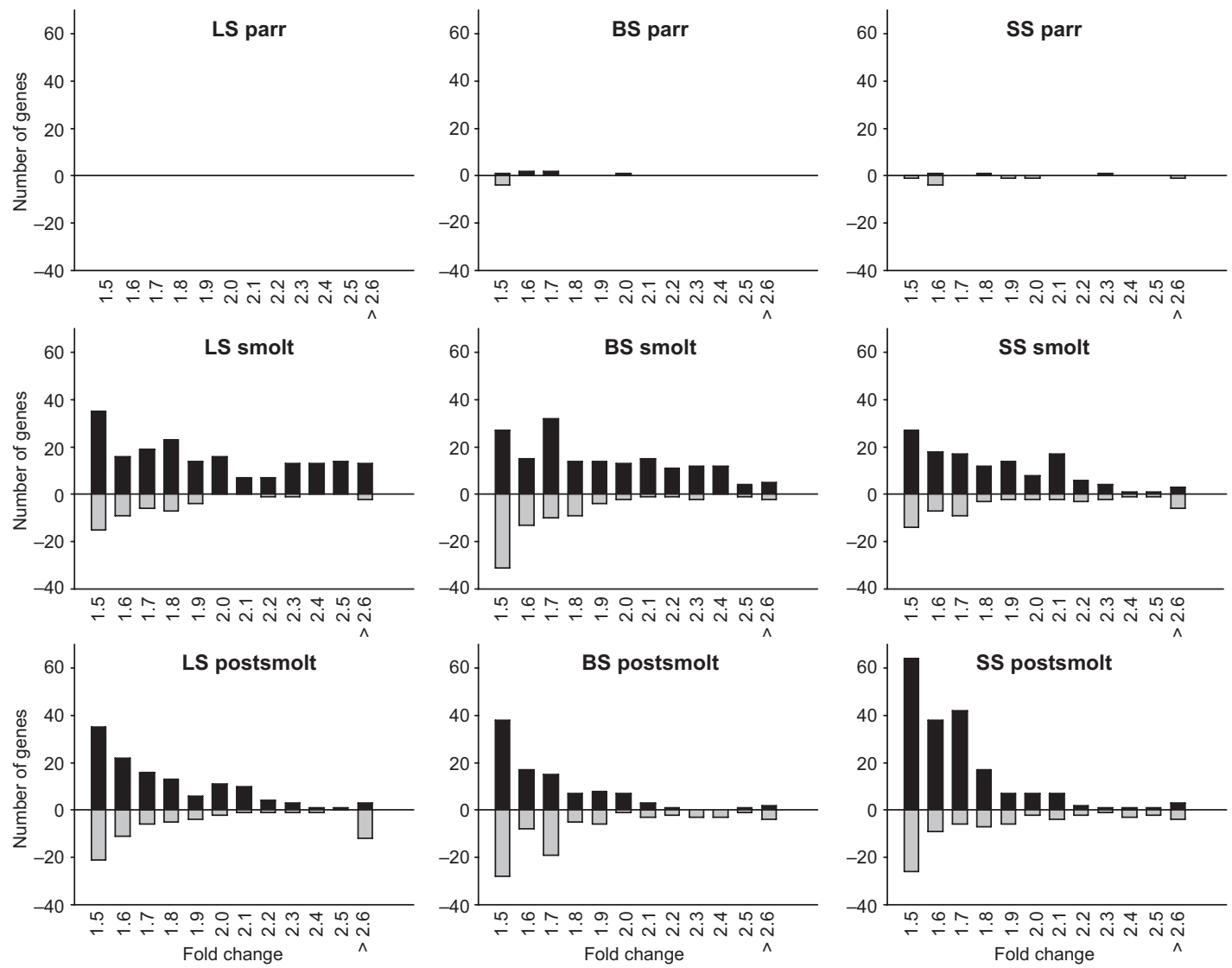

Fig. 3. Gene expression fold changes in the gill filaments of the Atlantic salmon (Salmo salar) in parr (top), smolt (middle) and postsmolt (bottom) phase of development. All comparisons are made relative to the gene expression in the parr fish of the landlocked stock. Up- and down-regulation of genes with a statistical significance (FDRadjusted $p \leq 0.1$, LIMMA "topTable" function) are indicated. LS = landlocked stock, BS = brackish-water stock, SS = seawater stock.

first-strand cDNA was synthesized and qPCR was performed using the DyNAmo ${ }^{\mathrm{TM}} \mathrm{SYBR}^{\circledR}$ Green 2-Step qRT-PCR Kit (Finnzymes, Espoo, Finland) and a DNA Engine ${ }^{\circledR}$ thermal cycler supplied with a Chromo4 Continuous Fluorescence Detector (MJ Research, Waltham, MA). Reaction conditions were as follows: $94{ }^{\circ} \mathrm{C}$ for 15 min, 40 cycles of $94{ }^{\circ} \mathrm{C}$ for $10 \mathrm{~s}, 57^{\circ} \mathrm{C}$ for $20 \mathrm{~s}$, and $72{ }^{\circ} \mathrm{C}$ for $30 \mathrm{~s}$. After PCR, the amplification of specific products was monitored by melting curve analysis. All analyses were made on all three salmon stocks on three different sampling dates, and were run in three technical replicates. qPCR methods were followed according to the Minimum Information for publication of Quantitative Real-Time PCR Experiments.

\section{Results}

\section{The extent of gene expression changes}

In all three salmon stocks, transformation of the young freshwater parr to the seawater adapted smolt and further to the postsmolt fish was associated with profound changes in gene expression of the gill at the transcript level (Fig. 3). In the parr phase, there were only few differences in gene expression between the three stocks (all comparisons are relative to the parr of LS). In BS, 1 gene (CA053105) was upregulated ( $>2$-fold change, a false discovery rate (FDR) adjusted $p<0.1$ ), and no genes were down-regulated in comparison with the LS parr. 
Fig. 4. Comparison of up- and down-regulated genes among developmental phases. A Venn diagram indicating the number of common/different genes, which were more than 2-fold either up- or downregulated $(p<0.1$, LIMMA "decideTests" function) in the parr, smolt and postsmolt fish of each stock. All numbers are relative to the gene expression in the parr of the LS stock. LS = landlocked stock, BS = brackish-water stock, SS = seawater stock.

\section{Up-regulated genes}

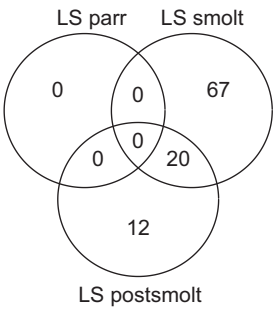

Down-regulated genes

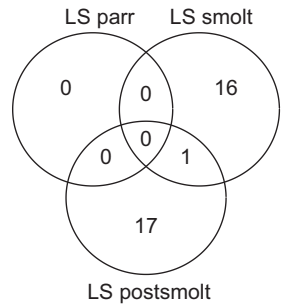

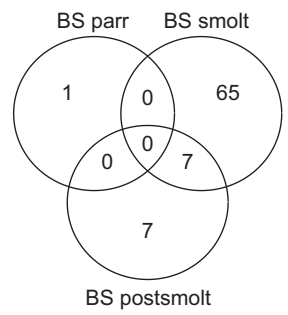

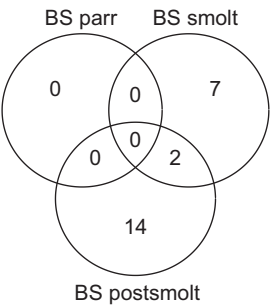

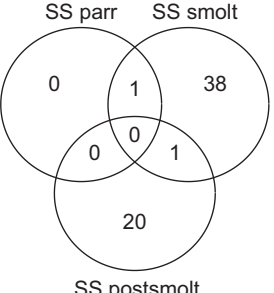

SS postsmolt

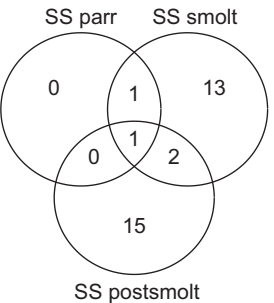

The number of up- and down-regulated genes in SS was 1 (CA053105) and 2 (CA050554, CA039292), respectively. Although the latter three genes are not annotated by GRASP, in a blastn search they show similarity to Tripartite motif-containing protein 25, SAM domain and HD domain-containing protein 1 and Zymogen granule membrane protein 16 precursor, respectively.

A broad change in gene expression occurred both in the transformation from parr to smolt and from smolt to postsmolt. In comparison to the parr fish of LS, the numbers of up-regulated genes in smolts were 87,72 and 40 for LS, BS and SS, respectively. The numbers of downregulated genes were substantially lower, being 17, 9 and 17 for LS, BS and SS, respectively.

In the postsmolt fish, the total number of differentially expressed genes was only 58\% (71) of that in the smolt phase (122). In the postsmolts, the numbers of differently expressed genes were 50, 30 and 39 for LS, BS and SS, respectively, from which $36 \%, 53 \%$ and $46 \%$ were down-regulated (relative to the LS parr). In LS, 20 genes (20.2\%) from a total of 99 upregulated genes were common for both the smolt and the postsmolt fish (Fig. 4). The percentages of the common up-regulated genes in BS and SS were $8.9 \%$ and $1.7 \%$, respectively. For downregulated genes, the portion of common genes

for the smolt and postsmolt fish varied between $2.9 \%$ and $9.4 \%$. Thus, in regard to the number of differentially expressed genes ( $>2$-fold difference) the postsmolt fish differ from the smolt fish less than the smolt fish differ from the parr fish. However, when all differentially expressed genes (a FDR-adjusted $p<0.1$ ) are taken into account (Table 1), the Euclidean distance values between the postsmolt fish and the smolt fish is about $11 \%$ higher than between the smolt and the parr. Collectively, these findings indicate that the parr-smolt and smolt-postsmolt transformations are associated with a broad change in gene expression of the gill.

\section{Patterns of gene expression changes}

The changes in gene expression can be divided into four main categories according to the pattern of transcript levels in different developmental phases. The largest group of differentially-expressed genes (36\%) showed an inverted V-type pattern of expression, i.e. the maximum expression was in the smolt phase with lower values both in parr and postsmolt fish (Fig. 5a). Genes encoding for $\alpha 1$ and $\beta 233$ subunits of the Na-pump and the majority of other ion transport genes including SERCA1 belong to this group (Table 2 and Fig. 6b). Several sequences 

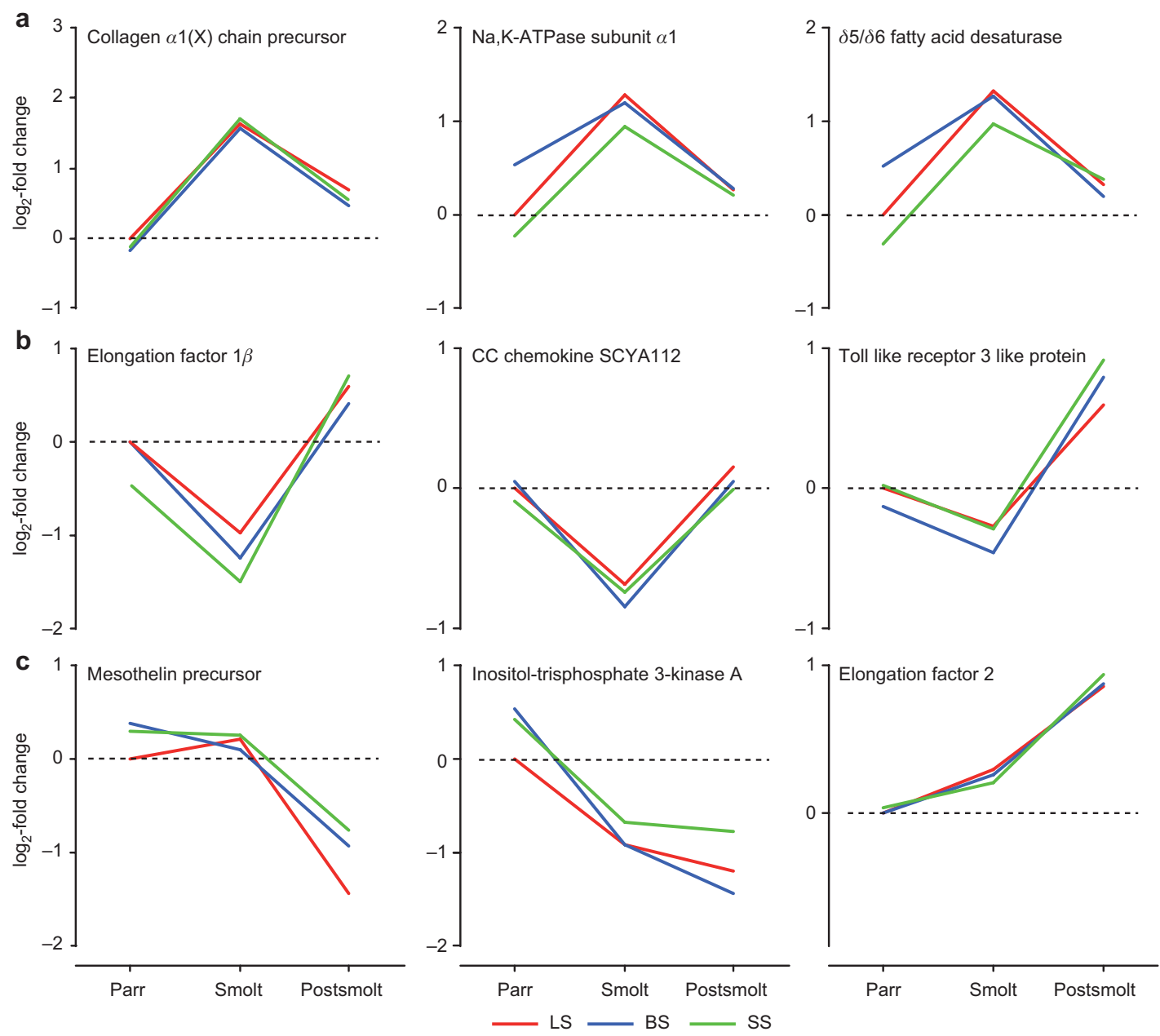

Fig. 5. Developmental patterns of gene expression changes. Gene expression in the gill filaments of the Atlantic salmon (Salmo salar) was grouped into four main categories according to the pattern of changes during the development. Majority of genes had either maximum or minimum expression in the smolt phase (a, b). Several genes had maximum expression in the postsmolt phase and fewer genes at the parr stage (c). Representative examples for each category are shown. Some genes belonging to these categories are listed in Table 2. LS = landlocked stock, BS = brackish-water stock, SS = seawater stock.

Table 1. Similarity in gene expression in three stocks as indicated by Euclidean distances between samples. The genes with FDR-adjusted $p \leq 0.1$ (LIMMA "topTable" function) were used in the analysis. LS = landlocked stock, BS = brackish-water stock, SS = seawater stock.

\begin{tabular}{lrrrrrrrr}
\hline Samples & $\begin{array}{c}\text { LS } \\
\text { parr }\end{array}$ & $\begin{array}{c}\text { BS } \\
\text { parr }\end{array}$ & $\begin{array}{c}\text { SS } \\
\text { parr }\end{array}$ & $\begin{array}{c}\text { LS } \\
\text { smolt }\end{array}$ & $\begin{array}{c}\text { BS } \\
\text { smolt }\end{array}$ & $\begin{array}{c}\text { SS } \\
\text { smolt }\end{array}$ & $\begin{array}{c}\text { LS } \\
\text { postsmolt }\end{array}$ & $\begin{array}{c}\text { BS } \\
\text { postsmolt }\end{array}$ \\
\hline BS parr & 7.3 & & & & & & & \\
SS parr & 8.2 & 9.9 & & & & & & \\
LS smolt & 18.5 & 17.5 & 20.8 & & & & & \\
BS smolt & 18.6 & 17.4 & 20.1 & 4.0 & & & & \\
SS smolt & 16.7 & 15.8 & 17.7 & 7.5 & 7.3 & & & \\
LS postsmolt & 17.4 & 18.8 & 20.1 & 19.4 & 20.1 & 19.5 & & \\
BS postsmolt & 16.6 & 17.5 & 18.2 & 18.8 & 18.9 & 18.9 & 7.5 & 11.5 \\
SS postsmolt & 18.9 & 20.5 & 21.5 & 21.9 & 23.1 & 21.2 & 9.7 & \\
\hline
\end{tabular}



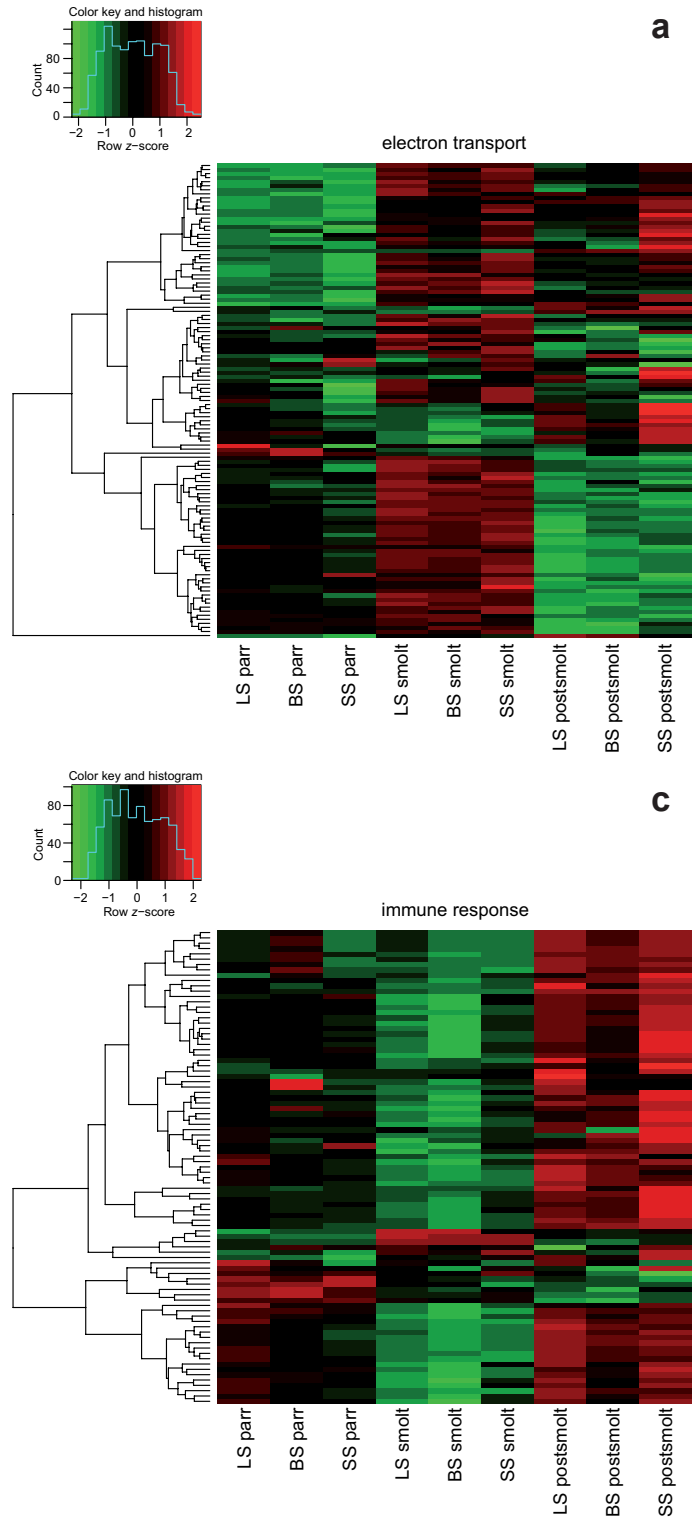

involved in a collagen synthesis - including genes for the collagen $\alpha 1, \alpha 2$ and $\alpha 3$ chains were particularly clearly enhanced in smolts. A third group of genes which were strongly up-regulated in smolts was formed by genes involved in aerobic energy metabolism of mitochondria including different subunits of cytochrome $c$ and ATP synthase (Fig. 6a). Also actin and genes involved in actin-related molecular structures like tropomyosin $\alpha 1$, fibronectin, SPARC precursor, forming-like protein-1 and myosin binding protein-H were up-regulated in the smolt phase.

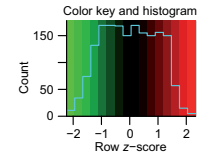

b

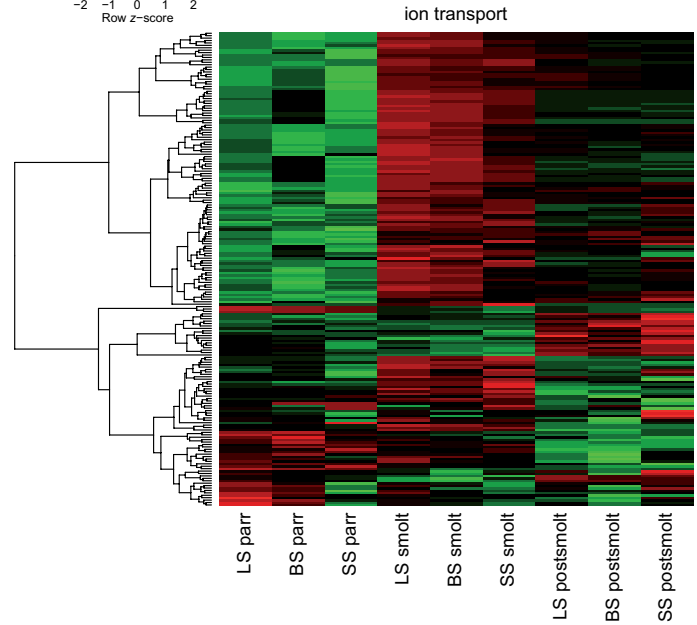

Fig. 6. Gene expression changes in three gene groups. Heat maps showing gene expression changes (with FDR-adjusted $p \leq 0.1$, LIMMA "topTable" function) in smoltification and desmoltification of the Atlantic salmon (Salmo salar) in three functional gene groups: (a) electron transport, (b) ion transport, and (c) immune response. LS = landlocked stock, BS = brackish-water stock, SS = seawater stock. The colour key indicates relative transcript expression on a $\log _{2}$-transformed scale.

The second largest group of genes (34\%) followed a V-type pattern of expression with the minimum expression level in smolts (Fig. 5b) (Table 2). Elongation factor $1-\beta$, peroxisomal multifunctional enzyme type 2 and proteasome activator complex subunit 2 are representative examples for this group. A major group of genes that show depressed expression in the smolt phase was formed by immunological defense genes (Fig. 6c). Several genes involved in innate and specific immunity including Toll-like receptor 3-like protein, MHC class I protein and $\mathrm{H} 2$ 


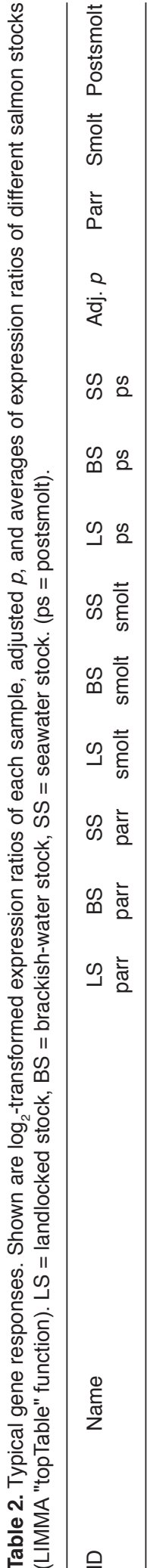

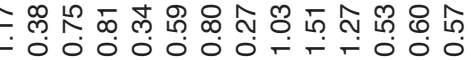

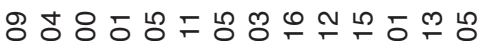

o ó

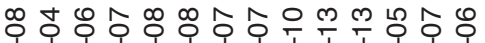

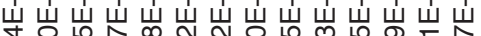
ก

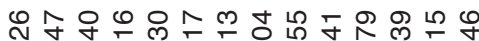

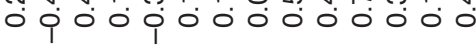

㐫

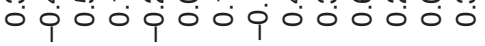

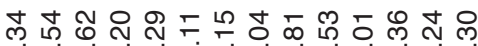
o

ㅇำ - $00000000 \div-000$

กุ่ - 0000000 - 0000

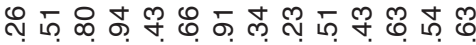
- $0000000 \%$

กิ

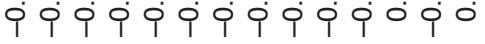
预

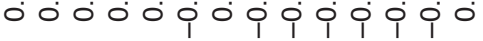

৪ ৪ ৪ ৪ ৪ ৪ ৪ ৪ ৪ ৪ ৪

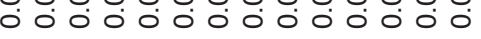

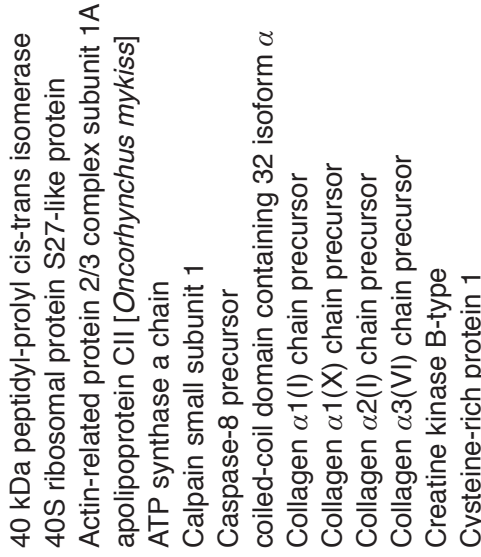

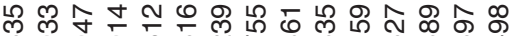

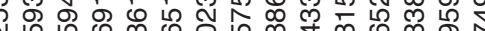
寸 ؛ ⿺辶寸

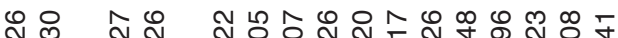

○. 00 0 00000000000

ํํ

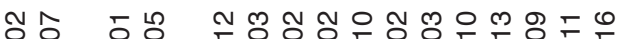

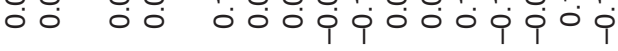

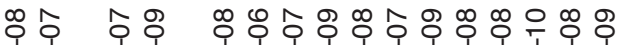

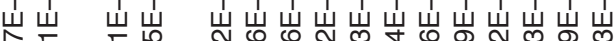
लं

సิ

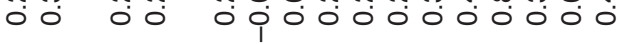

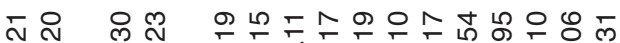

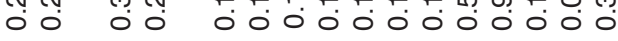

이 m

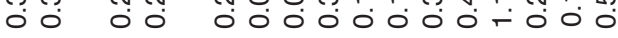

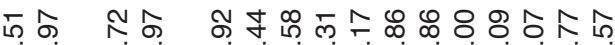

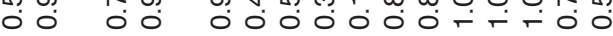

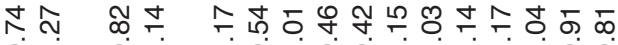
O- O-

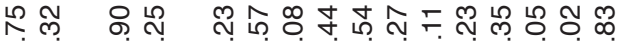

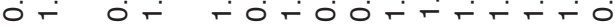

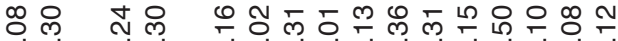

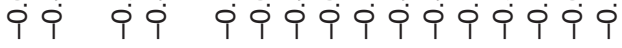

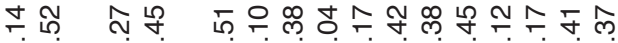

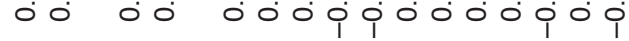

ㅇㅇ ㅇㅇㅇㅇㅇㅇㅇ ৪ ㅇㅇㅇ O
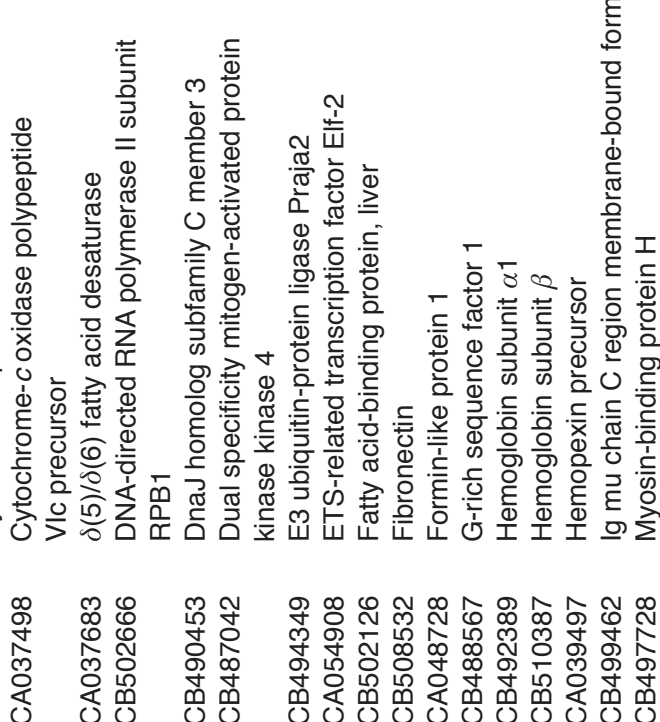
โ 

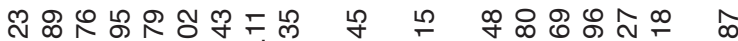

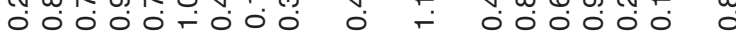

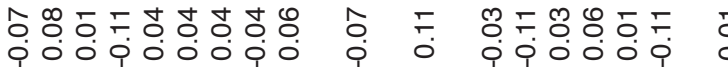

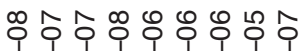

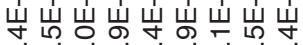

\section{ᄂ

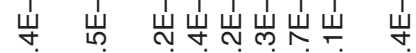

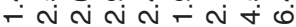

เิ

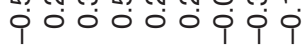

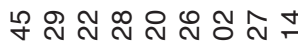

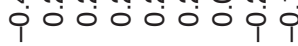

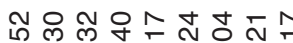

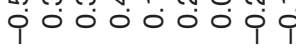

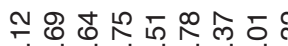
000000000

กิ ลิ่

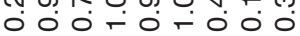

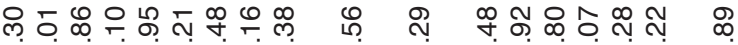

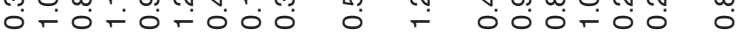

กำ ipipipipi o i óipip i

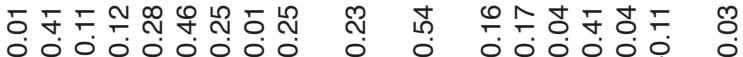
०00,00000

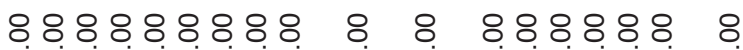

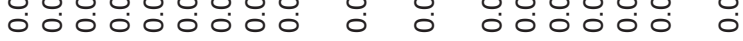

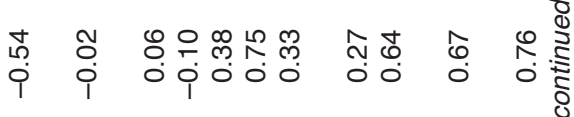

ช

i

ป

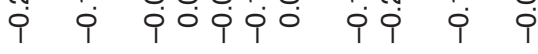

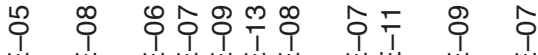

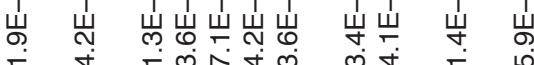

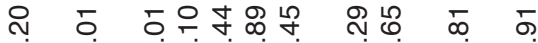

O 0 i

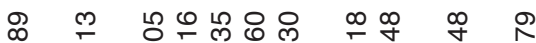

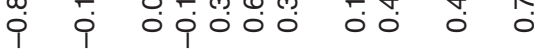

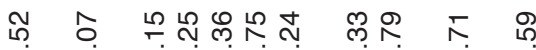

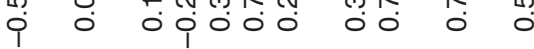

๒

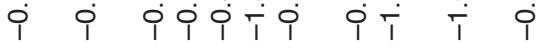

น

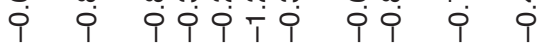

๓

i i ípió î́ i i

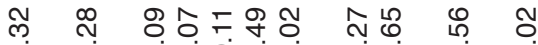

i i ioipi î i o

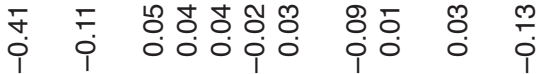

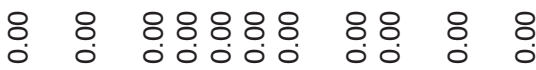
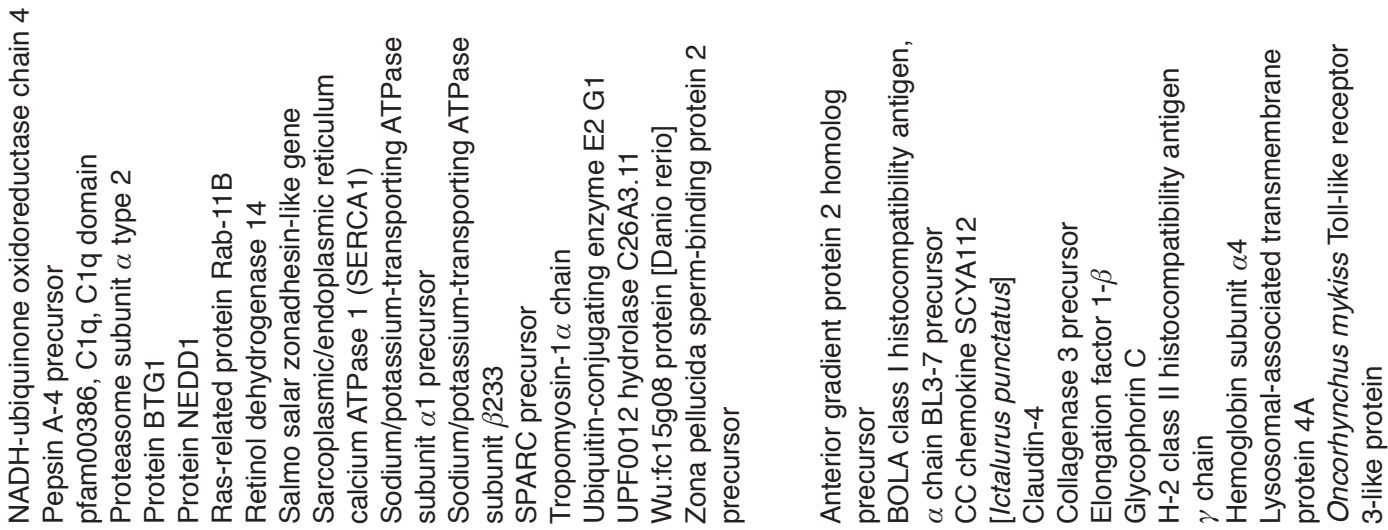

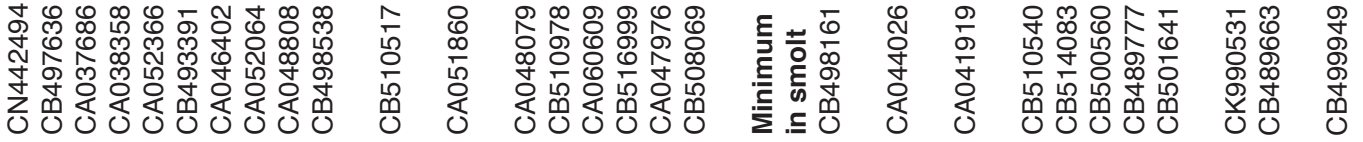




\begin{tabular}{|c|c|c|c|c|c|c|c|c|}
\hline$\frac{\frac{E}{0}}{\frac{5}{0 D}}$ & 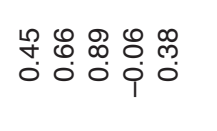 & $\begin{array}{l}8 \\
0 \\
0\end{array}$ & 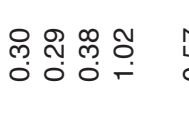 & 占 & 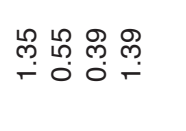 & 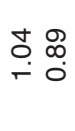 & 茴 & 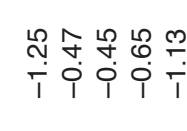 \\
\hline ஸे & 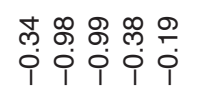 & $\begin{array}{l}\infty \\
0 \\
0 \\
9\end{array}$ & 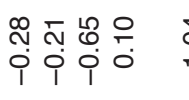 & 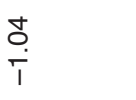 & 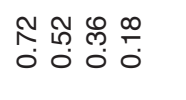 & 宅 & 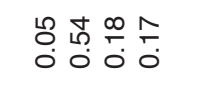 & 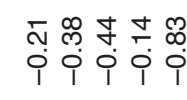 \\
\hline$\frac{\bar{L}}{0}$ & 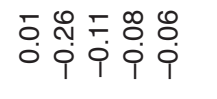 & 우 & 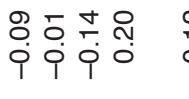 & $\stackrel{N}{\stackrel{i}{i}}$ & $\begin{array}{llll} & 0 & 0 & 0 \\
0 & 0 & 0 \\
0 & 0 & 0 & 0 \\
1 & 1 & 0\end{array}$ & $\stackrel{1}{L}$ & 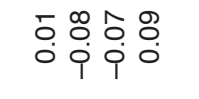 & 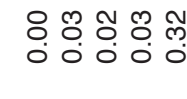 \\
\hline$\frac{Q}{\dot{\bar{\pi}}}$ & 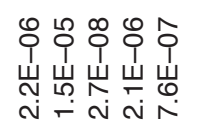 & 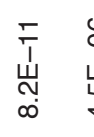 & 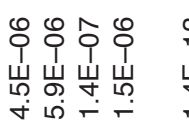 & 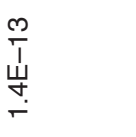 & 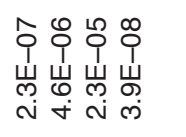 & 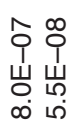 & 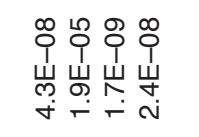 & 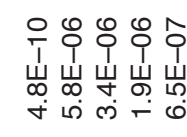 \\
\hline ஹ & 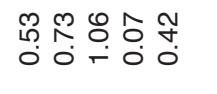 & $\begin{array}{l}\stackrel{0}{0} \\
0\end{array}$ & 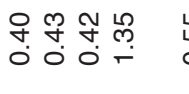 & 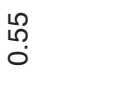 & 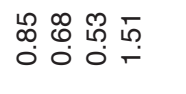 & 孛 & 끔 욤 & 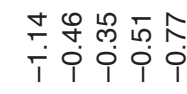 \\
\hline ஜூ & 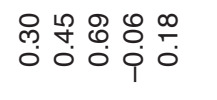 & $\begin{array}{l}\infty \\
\stackrel{0}{0} \\
0\end{array}$ & \begin{tabular}{l}
0 \\
\hdashline \\
\hdashline \\
0
\end{tabular} & 点 & 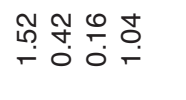 & 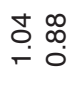 & 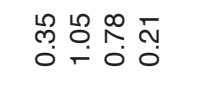 & 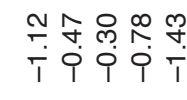 \\
\hline c) & 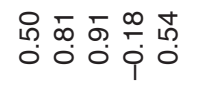 & $\begin{array}{l}\infty \\
\stackrel{0}{0} \\
0\end{array}$ & 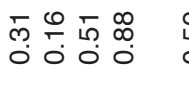 & 茴 & به & 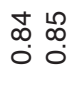 & 年옹음 & 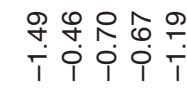 \\
\hline ळ & 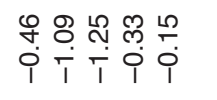 & $\begin{array}{l}\infty \\
\infty \\
\stackrel{9}{1}\end{array}$ & 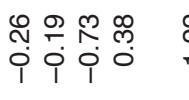 & $\frac{\text { N̦ }}{T}$ & 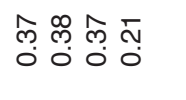 & ભ̊ & $\begin{array}{l}\infty \\
\\
0\end{array}$ & 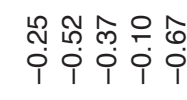 \\
\hline 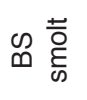 & 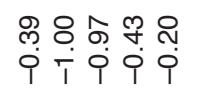 & $\begin{array}{l}\stackrel{0}{0} \\
\stackrel{9}{9}\end{array}$ & 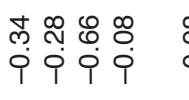 & $\begin{array}{l}\infty \\
\stackrel{\infty}{9} \\
0 \\
\end{array}$ & 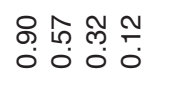 & 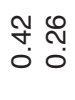 & 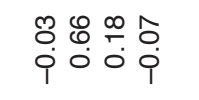 & 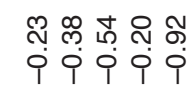 \\
\hline$\omega \frac{5}{\frac{t}{0}}$ & 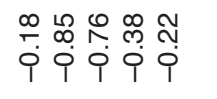 & $\begin{array}{l}100 \\
0 \\
0\end{array}$ & 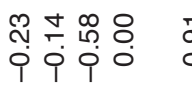 & $\begin{array}{l}\text { - } \\
\text { ọ } \\
1\end{array}$ & 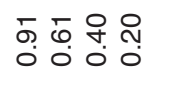 & 宅 & $\begin{array}{llll}- & 0 & 0 & 0 \\
0 & 0 & 0 \\
0 & 0 & 0 & 0 \\
1 & 0\end{array}$ & 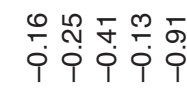 \\
\hline ભ 㐫 & 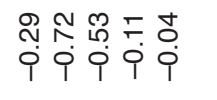 & $\begin{array}{c}\hat{N} \\
\text { ị }\end{array}$ & 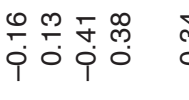 & 芦 & 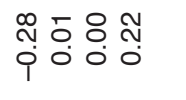 & $\begin{array}{l}0.0 \\
0 \\
0\end{array}$ & 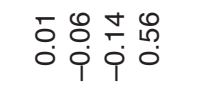 & 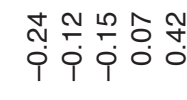 \\
\hline 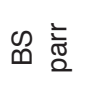 & 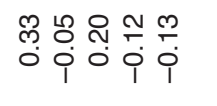 & 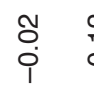 & 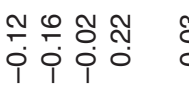 & 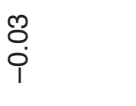 & $\begin{array}{l}\infty \\
0 \\
0 \\
0\end{array}$ & $\begin{array}{ll}8 & 8 \\
0 & 0\end{array}$ & 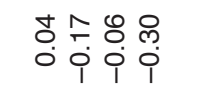 & 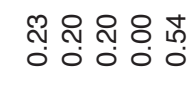 \\
\hline 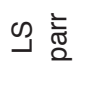 & $\begin{array}{l}8.88 .80 \\
\circ 0 \\
\end{array}$ & \& & 888.8 & \&: & $\begin{array}{l}8.8 \% 8 \\
000\end{array}$ & 8: & $\begin{array}{l}8.8 \% 8 \\
0000\end{array}$ & 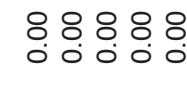 \\
\hline $\begin{array}{l}\text { E } \\
\text { त्ञ } \\
Z\end{array}$ & 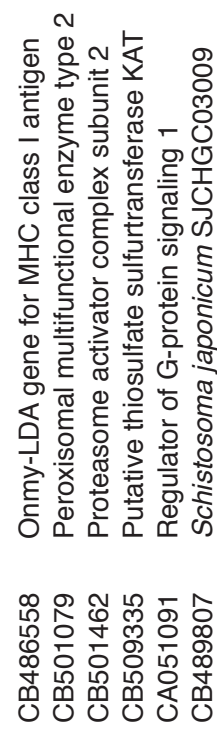 & 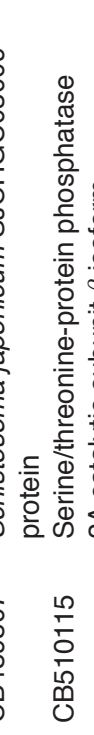 & 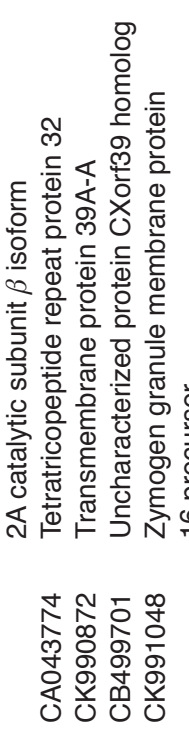 & 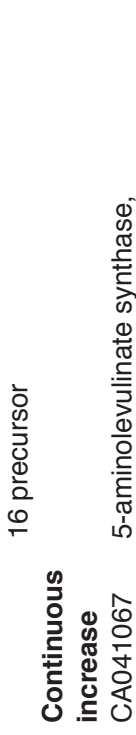 & 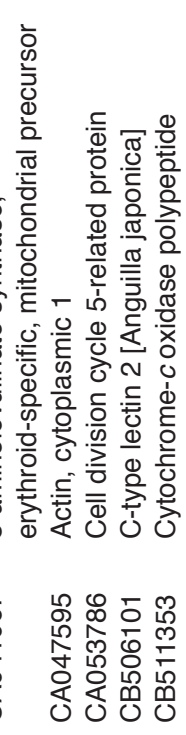 & 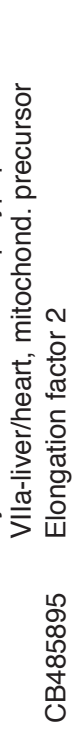 & 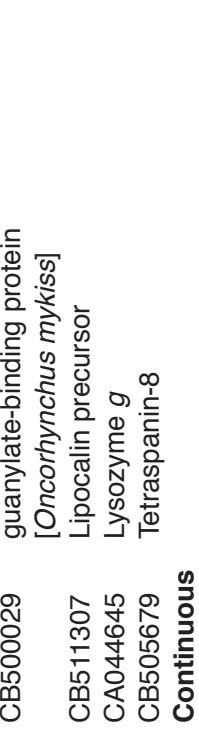 & 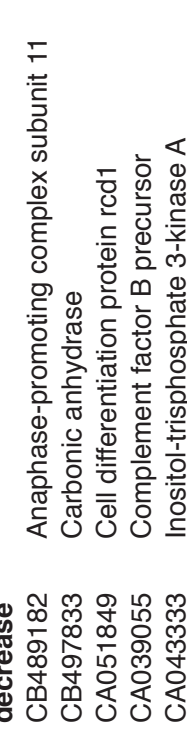 \\
\hline
\end{tabular}




\begin{tabular}{|c|c|c|c|c|c|}
\hline 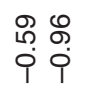 & $\begin{array}{l}\stackrel{2}{R} \\
\stackrel{9}{i}\end{array}$ & 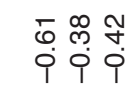 & & $\begin{array}{l}\text { ô } \\
0\end{array}$ & 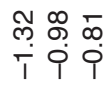 \\
\hline 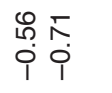 & $\begin{array}{l}\infty \\
0 \\
0 \\
i\end{array}$ & 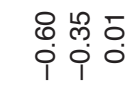 & & $\begin{array}{l}0 \\
0 \\
0\end{array}$ & 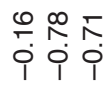 \\
\hline 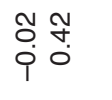 & F & 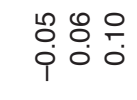 & & 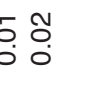 & 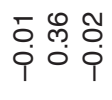 \\
\hline 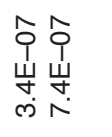 & 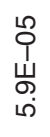 & 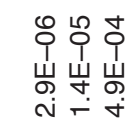 & & $\begin{array}{l}0 \\
1 \\
1 \\
1 \\
0\end{array}$ & 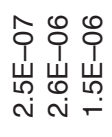 \\
\hline 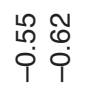 & $\begin{array}{l}\hat{0} \\
0 \\
i\end{array}$ & 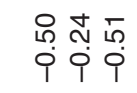 & & $\begin{array}{l}0 \\
0 \\
0 \\
\end{array}$ & 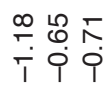 \\
\hline 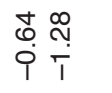 & \begin{tabular}{l}
\multirow{0}{*}{} \\
$\infty$ \\
$i$
\end{tabular} & 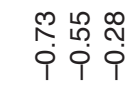 & & $\begin{array}{l}0 \\
0 \\
0 \\
1\end{array}$ & 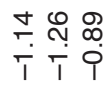 \\
\hline 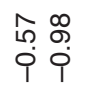 & $\begin{array}{l}\stackrel{2}{2} \\
0 \\
i\end{array}$ & 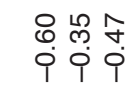 & & $\begin{array}{ll}n & 0 \\
0 & 0 \\
0 & 0 \\
1 & 1 \\
1\end{array}$ & 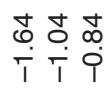 \\
\hline 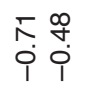 & $\begin{array}{l}\text { ๙ิ } \\
\text { ధं }\end{array}$ & 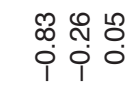 & & 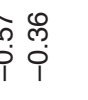 & 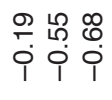 \\
\hline 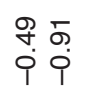 & $\begin{array}{l}\overline{0} \\
\dot{\varphi}\end{array}$ & 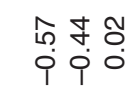 & & 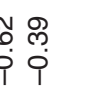 & 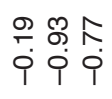 \\
\hline $\begin{array}{l}\text { 足 } \\
\text { 足 } \\
\text { i }\end{array}$ & $\begin{array}{l}\bar{n} \\
0 \\
i\end{array}$ & 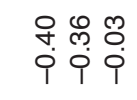 & & 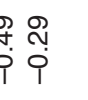 & \begin{tabular}{lll}
0 & 0 \\
\hdashline & 0 \\
\hdashline & 0 \\
1 & 1 & 0 \\
\end{tabular} \\
\hline $\begin{array}{l}0 \\
0\end{array}$ & F & 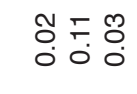 & & ơ & 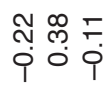 \\
\hline $\begin{array}{l}0 \\
\frac{1}{0} \\
0\end{array}$ & $\begin{array}{l}\text { ָ̃ } \\
\text { ฺ }\end{array}$ & 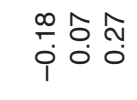 & & 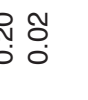 & $\begin{array}{lll}\infty & R \\
& 0 \\
0 & 0 & 0 \\
0\end{array}$ \\
\hline $\begin{array}{l}80 \\
8 \\
0\end{array}$ & \& & $\begin{array}{l}8.8 \\
0 \\
\end{array}$ & & '0 & $\begin{array}{l}8 \\
\end{array}$ \\
\hline 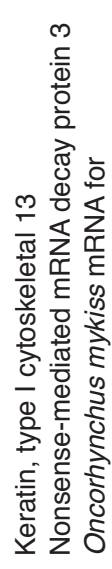 & & 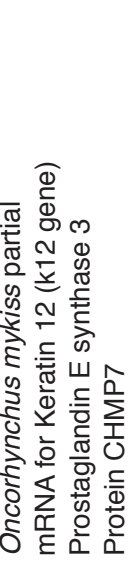 & 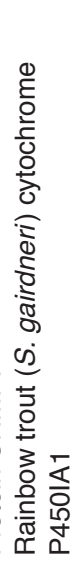 & 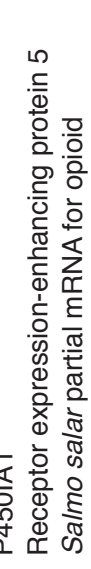 & 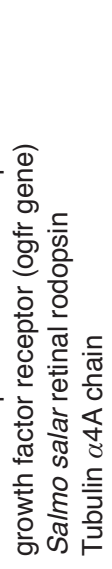 \\
\hline 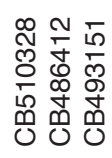 & & 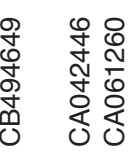 & $\begin{array}{l}8 \\
0 \\
o \\
o \\
0 \\
0\end{array}$ & $\begin{array}{l}\overline{1} \\
0 \\
0 \\
0 \\
0 \\
0 \\
0 \\
0 \\
0 \\
0\end{array}$ & 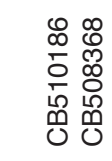 \\
\hline
\end{tabular}

class II histocompatibility antigen were downregulated in the smolt phase. There were also several non-annotated genes that show either $\mathrm{V}$-pattern or inverted V-pattern expression. Noticeably, the $\mathrm{V}$ and inverted $\mathrm{V}$ patterns are not always completely symmetric in shape suggesting that the gene expression changes associated with the parr-smolt transition are either only partly reversed or somewhat enhanced in the postsmolt fish. This appears as an aggregation of differentially expressed genes in the lower fold change values in the postsmolt fish (Fig. 3, bottom). This is also evident in Table 1; the parr fish differ from the postsmolt fish even more than from the smolt fish.

A smaller number of genes showed relatively little changes between parr and smolt phases but were either up- or down-regulated in the postsmolt phase (Fig. 5c). The former group includes genes involved in immunodefense, while the latter includes enzymes of energy metabolism. A few genes showed either continuous increase or decline throughout the whole development process from parr to postsmolt fish (Fig. 5c).

\section{Comparison of gene expression between salmon stocks}

The changes in gill gene expression in the three salmon stocks are strikingly similar considering the widely different habitats to which the salmon smolts would migrate under natural conditions. Among the smolt fish, $25.8 \%$ of the down-regulated genes ( $>2$-fold change, a FDR-adjusted $p<0.1)$ and $79.1 \%$ of the up-regulated genes are the same in at least two of the stocks. In the postsmolt fish, the percentages of common genes are $55.6 \%$ and $40.9 \%$ for down- and up-regulated genes, respectively. If all differentially expressed genes are taken into account, the similarity is even more striking (Fig. 7). The similarity of gene expression applies not only to the genes expressed but also to the extent of the gene expression changes (Figs. 3 and 7). Nevertheless, there are some minor differences between the salmon stocks which appear in the pattern of how closely the three stocks are related to each other at different developmental stages. In the cluster analysis, the parr-phase LS is grouped 

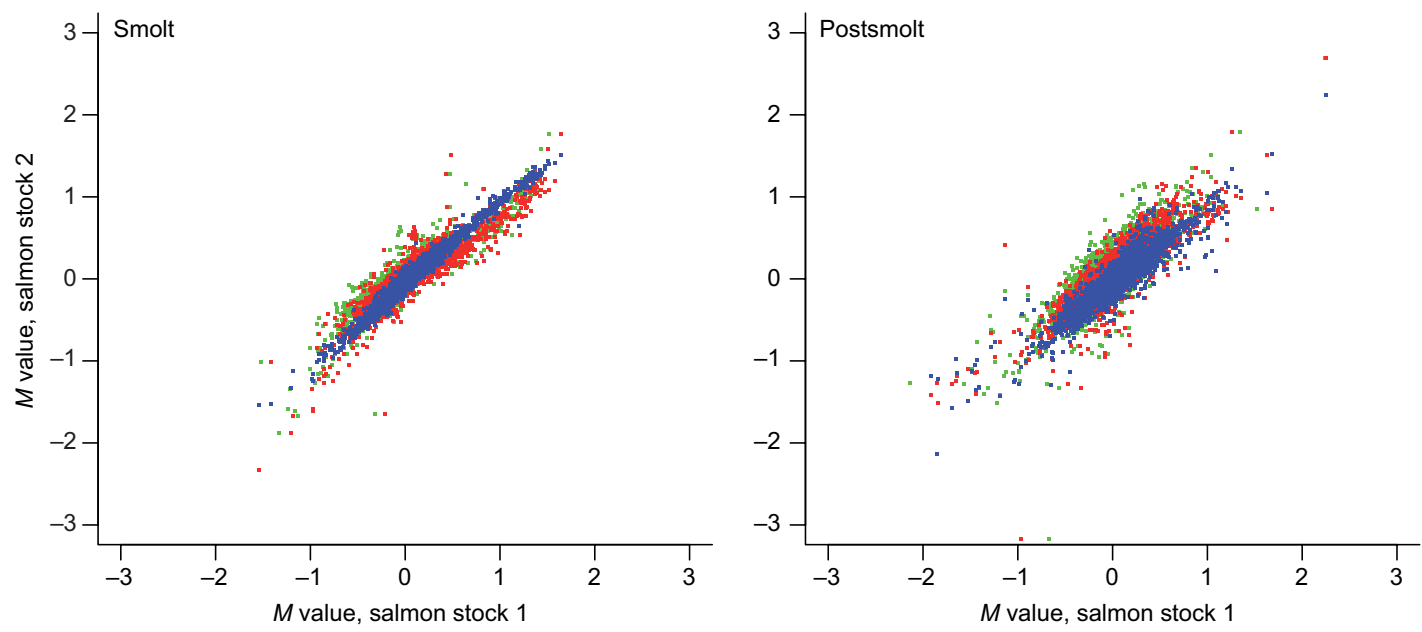

$$
\text { - LS/BS " LS/SS "BS/SS }
$$

Fig. 7. Similarity of gene expression changes in the three salmon stocks. A scatterplot of the $M$ values (log ${ }_{2}$-fold change) of differentially expressed genes (FDR-adjusted $p \leq 0.1$ ) among the different salmon stocks at the smolt or postsmolt stage. At the smolt stage the correlation coefficients are $0.98,0.92$ and 0.92 for LS/BS, LS/SS and BS/ SS, respectively. At the postsmolt stage, the correlation coefficients are $0.91,0.86$ and 0.82 for LS/BS, LS/SS and BS/SS, respectively. LS = landlocked stock, BS = brackish-water stock, SS = seawater stock.

together either with the parr-phase BS or SS, depending on the genes used in the analysis. In the smolt and postsmolt phase, LS and BS are grouped together and separated from SS (Fig. 8). In the smolt fish, the differences among the stocks in gene expression (Euclidean distance) are smaller than in either the parr or postsmolt phase of development, and the differences among stocks are the greatest in the postsmolt phase (Table 1).

\section{Validation of microarray analysis}

To verify the altered gene expression detected by the microarray, we analyzed a subset of 10 target genes using quantitative real time PCR. There was a good correlation of the array and real time data with 7 genes (Na,K-ATPase, $\alpha 1$; collagen $1(\mathrm{x}) ; 5$-aminolevulinate synthase; elongation factor $1 \beta$; C-type lectin 2 ; mesothelin precursor; Lysosomal-associated transmembrane protein) representing different developmental patterns and functional groups (Fig. 9). In the case of 2 genes, the correlation was partial (anaphase-promoting complex subunit 11; MAPKK) and for 1 gene (Formin-like protein) the developmental pattern was opposite in the array and real time analyses. High similarity $(\sim 70 \%)$ of the array

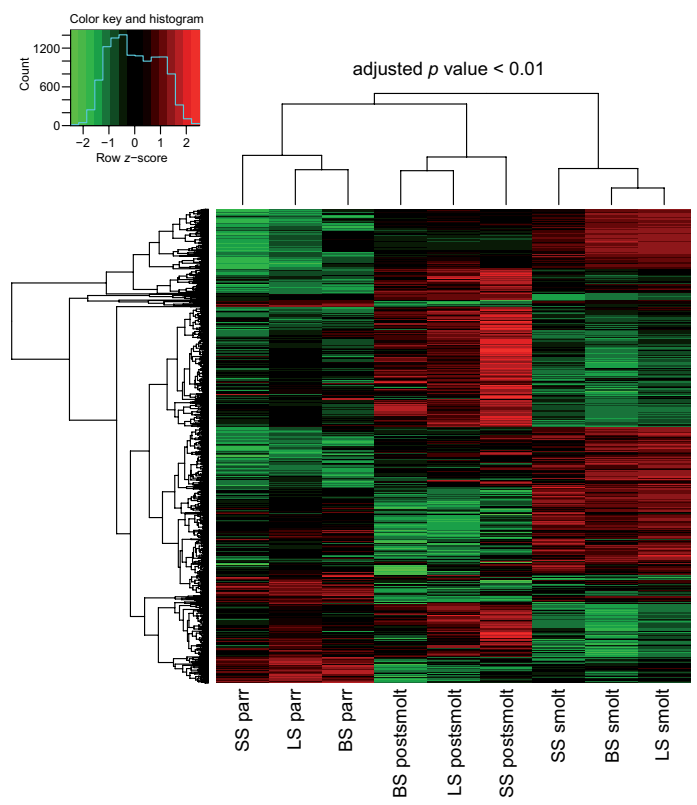

Fig. 8. A heat map of stock-related differences in gene expression. Gene expression of the gill filaments during the parr-smolt transformation and desmoltification of the Atlantic salmon. Top genes (LIMMA "topTable" function) which were either up- or down-regulated (FDR-adjusted $p \leq 0.1)$. LS = landlocked stock, BS = brackish-water stock, SS = seawater stock. The color key indicates relative transcript expression on a $\log _{2}-$ transformed scale. 

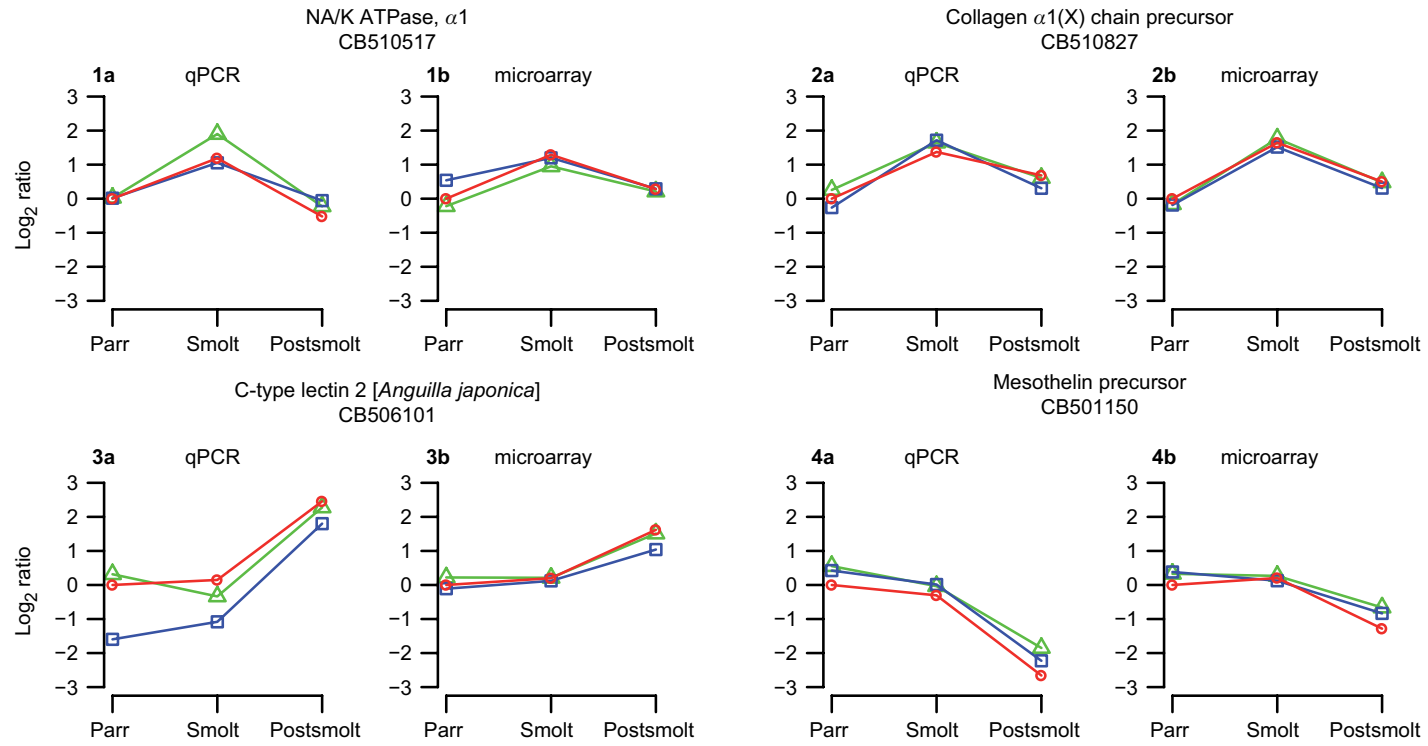

5-aminolevulinate synthase, erythroid CA041067
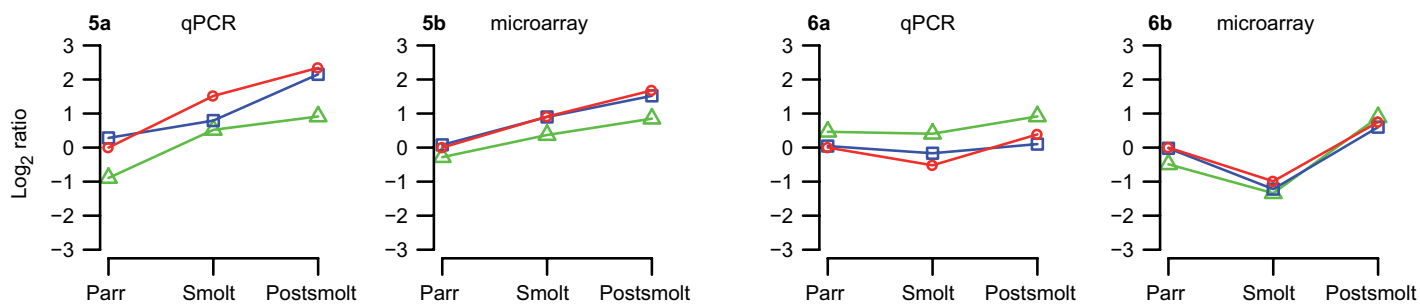

Lysosomal-associated transmembr. prot. 4A CB489663

Anaphase-promoting complex subunit 11 CB489182
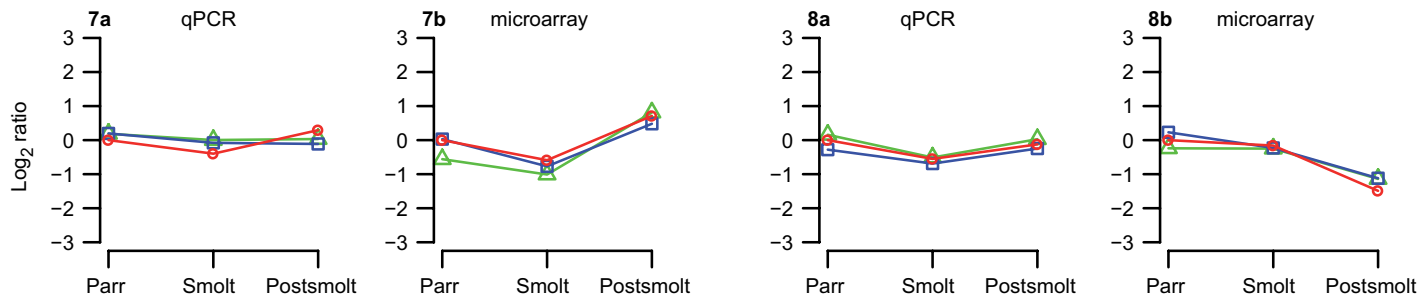

MAPKK

CB487042
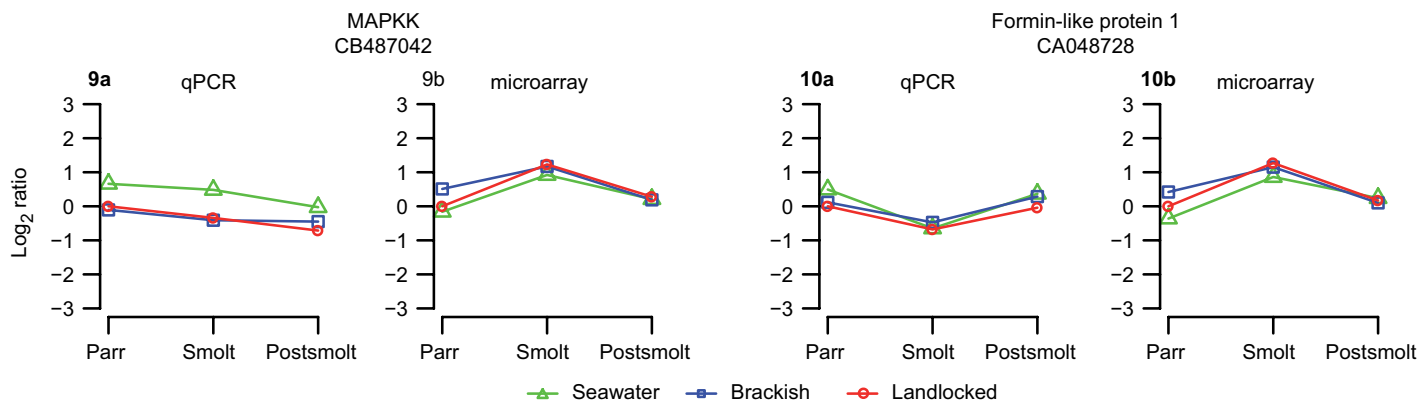

$\triangle$ Seawater $\rightarrow$ Brackish $\multimap$ Landlocked

Fig. 9. The expression ratios of the 10 genes obtained from the qPCR analysis compared with the results obtained from the microarray analysis. 
and real time data indicates that the GRASP $16 \mathrm{~K}$ cDNA salmonid microarray (von Schalburg et al. 2005) works well for the developing salmonid fish.

\section{Discussion}

The present high-throughput analysis shows that smoltification of the Atlantic salmon is associated with extensive and partly reversible changes in gene expression of the major ion-regulatory organ, the gills. In essence, the changes in gene expression are both qualitatively and quantitatively similar in the three studied salmon stocks irrespective of the salinity level of their natural habitat where they would migrate as smolts. Even LS, which has been separated from seawater entry for over 5000 years (Berg 1985), and completes its whole life-cycle in the freshwater environment, goes through similar and extensive changes in gene expression as SS that migrates from the Teno River to the full-strength seawater of the Arctic Ocean. This is contrary to the hypothesis that developmental changes in gene expression of the gill are markedly less developed in the landlocked salmonid populations in comparison with those in seawater migrating anadromous salmon stocks (Barbour \& Garside 1983, Birt et al. 1991, Birt \& Green, 1993, Nilsen et al. 2003), but consistent with the findings that most features of the parr-smolt transformation appear to be retained in the developmental pattern of the lake-living non-anadromous salmonids including LS of Lake Saimaa (Chernitsky and Loenko 1983, Koch 1983, Burton \& Idler 1984, Schmitz 1995, Kiiskinen et al. 2002).

Many of the differentially-expressed genes showed either V-pattern or inverted V-pattern expression during the smoltification-desmoltification cycle suggesting that those genes were up- and down-regulated, respectively, in the smolt phase, and are therefore closely associated with the parr-smolt transformation. In addition to the $\alpha$ subunits of the $\mathrm{Na}, \mathrm{K}$-ATPase, which have been extensively characterized in salmonids (D'Cotta et al. 1996, Seidelin et al. 2001, Bystriansky et al. 2006, Nilsen et al. 2007), other genes associated with the ion and electron transport were up-regulated in smolts. Mitochon- drial genes involved in aerobic energy metabolism were up-regulated in smolts as expected on the basis of an increase in numbers and size of the mitochondria-rich chloride cells in the gill of the smolting Atlantic salmon (Langdon \& Thorpe 1985). Similar up-regulation of mitochondrial genes and Na,K-ATPase between the parr and smolt phases of development has recently been reported for this species by Seear et al. (2010). These changes suggest that mitochondrial activity and aerobic energy production reach their peak value in the smolt phase, and are significantly lower in both parrs and postsmolts. A concomitant increase in expression of the mitochondrial and ion transport genes probably reflects an increased energy demand of the hypoosmotic regulation and/or necessary preparatory changes for life in the hyperosmotic environment. These transcriptomic changes are very consistent with the previous physiological and molecular findings regarding gill function of the smolting salmonid fish (Hoar 1976, Folmar \& Dickhoff 1980, Langdon 1985), and indicate that the genetic basis of the parr-smolt transformation and hypoosmotic regulation by the gill is much broader than might have been anticipated, possibly involving other cell types in addition to the mitochondria-rich and pavement cells.

Interestingly, genes encoding collagen were strongly up-regulated in the smolt phase as noted also by Seear et al. (2010). Besides being a ubiquitous component of the intercellular matrix, collagen is an essential structural component of the pillar cells of the gill filaments. Pillar cells are specialized vascular cells with characteristics of both endothelial and smooth muscle cells which are situated perpendicular to the two cell layers of the gill filaments thereby regulating volume of the vascular space and blood flow within the secondary gill lamellae (Stensløkken et al. 1999). Pillar cells are traversed by $5-8$ bundles of collagen which are surrounded by infoldings of the pillar cell plasma membrane. Extracellular collagen columns are connected to the intracellular contractile machinery of actin, $\alpha$-actinin and myosin via integrin receptors and fibronectin (Kudo et al. 2007). Transcriptomic analysis showed that in addition to collagen fibronectin, actin, $\alpha$-actinin, actin-related protein 2/3 complex subunit, SPARC precursor, form- 
ing-like protein 1 , and myosin-binding protein $\mathrm{H}$ were up-regulated in the smolt phase. Several of these genes are also involved in contractile function of pillar cells and collagen columns (Kato et al. 2009). These findings strongly point to the possibility that in the smolt phase, pillar cell function is up-regulated possibly to ensure structural integrity and intact blood flow of the gill lamellae in response to changing osmotic stress (Mistry et al. 2004, Hyndman \& Evans 2009). The pillar-cell function is an interesting and unexplored area in the smolt physiology of the salmonid fish, and therefore the present findings provide a good starting point for hypothesisbased studies on the pillar-cell function in the parr-smolt transformation.

Some differentially-expressed genes showed their peak expression in the postsmolt phase. The majority of these genes are probably not directly associated with smoltification or desmoltification but rather with the changing environmental conditions, e.g. the rise of water temperature. Several genes involved in immunological defense belong to this group and might be required to meet the increasing challenge posed by pathological micro-organism in warming waters. The gill epithelium forms a barrier between the organism and the environment, and is harboured with molecules of innate and specific immune systems. Temperature rise enhances the immunodefense and is suggested to specifically enhance the specific immune system, while innate immunity may be less temperaturedependent (Bly \& Clem 1992, Alcorn et al. 2002). Even though there is a continuous rise of temperature during the development of the fish (Fig. 1), many of the genes involved in immunological defense showed their minimum expression in the smolt phase (Fig. 7c) and thus have a pattern of the mirror image to the genes of mitochondria, energy metabolism and ion transport. This is consistent with the previous findings that immunodefense is significantly depressed in the smolt phase (Rønneseth et al. 2005). The parrsmolt transformation is a hormonally controlled process in which cortisol plays an important role in transforming ion regulatory mechanisms from hyperosmotic to hypoosmotic (Folmar \& Dickhoff 1980, McCormick 1996, Mommsen et al. 1999). Although cortisol is probably vital for solving the hyperosmotic stress of the seawater entry, it is also an immunosuppressive hormone with negative consequences to the immunological defense mechanisms (Muona \& Soivio 1992, Espelid et al. 1996). Hence, at the smolt stage the fish may suffer from suppressed immunological protection possibly due to the cortisolmediated trade-off between energy allocation to ion regulation and immunological defense (McCormick \& Saunders 1987).

The parr-smolt transformation is an intense stress to salmon (as indicated by the increased cortisol levels) rendering them vulnerable to infections and increased predatory risks during and following the migration (Smail et al. 1992, Mesa et al. 1999). Energetic costs of transforming the ion excretory tissues and consequent reductions in survival rates are expected to impose selective pressures against the parr-smolt transformation unless it is associated with significant adaptive advantages. In the landlocked salmon strains, remodeling of the molecular machinery of the epithelial cells from a hyperosmoregulatory to a hypoosmoregulatory function can be seen as an energy-consuming process that results in trade-offs with other vital body functions like immunodefense. Hence, it would be expected that genetic mechanisms inducing or maintaining hypoosmoregulatory functions would be weaker or non-extant in the freshwater and brackish water resident salmon. Indeed an improper parr-smolt transformation appearing as weak salinity tolerance, low gill $\mathrm{Na}, \mathrm{K}$-ATPase activity or an atypical appearance of some other smolt characteristic has been reported for fish of some non-anadromous populations of the Atlantic salmon (Barbour \& Garside 1983, Burton \& Idler 1984, Sutterlin \& MacLean 1984, Birt et al. 1991, Birt \& Green, 1993, Nilsen et al. 2007), possibly due to weakened hormonal responses to environmental cues as a consequence of negative selection pressure on unnecessary traits of the smolt fish (McCormick et al. 2007). However, in other landlocked populations of the Atlantic salmon preparatory physiological adaptations are strongly expressed (Chernitsky \& Loenko 1983, Koch 1983, Burton \& Idler 1984, Schmitz 1995, Kiiskinen et al. 2002) which raises the question: Why is the extensive gene expression change of the parr-smolt transformation, typical for the 
anadromous salmon, retained in the landlocked populations? The simplest explanation is that 8000-10 000 years is too short a period to cause genetic differentiation of the landlocked salmon from their anadromous ancestors. However, the weakening of parr-smolt characteristics in some salmon populations indicates that selective pressure and number of generations have been sufficient to allow selection against smoltification. In those populations where the genomic basis for a full-strength parr-smolt transformation has been retained, the hypoosmoregulatory ability is probably linked to the behavioural component of migration and hence may function as a vital signaling system for migration from the relatively poor riverine environment to a more favourable growing habitat of the downstream lake (Staurnes et al. 1992). The stocks that have partly lost their parr-smolt characteristics often represent dwarfed salmon populations which may not obtain great benefit from migration since productivity levels of stream and lake habitats do not differ significantly; the benefits of migration are minimal which could have lead to weakening of the signaling function of smolting. Therefore, the results of this study are consistent with the idea that the genetic basis of the parr-smolt transformation is retained as a migration inducer in those landlocked salmon populations for which migration provides growth-favourable environment to the postsmolt fish. Indeed, the fish of LS grow much larger with an average body mass of 3.7 and $5.3 \mathrm{~kg}$ (and up to the length of $100 \mathrm{~cm}$; statistics of Game and Fisheries Research Institute in Finland for years $1980-2007, n=578$ ) at maturity for female and male, respectively, than the stunted salmon strains, e.g. the "blege" with the maximum size of $0.25 \mathrm{~kg}$ and $35 \mathrm{~cm}$ (Dahl 1928).

To reveal population-related differences in the genetic basis of smoltification, the gill tissue was pooled from two gill arches of 10 fish for each population at three time points of development. Collection of a large piece of tissue from a relatively large number of individual fish provides a representative sample of gene transcripts for population comparisons, while restricting the number of hybridization (30) and thereby keeping experimental costs reasonable. Pooling the samples also reduces the variation in gene expression. However, in the absence of biological replicates, an analysis of the pooled samples cannot provide information about the interindividual variation of gene expression. It is possible, at least theoretically, that stock-related differences exist between fish individuals in the process of smoltification, and these would have been lost in the absence of biological replicates and by pooling the samples. Whether the landlocked salmon stock includes individuals that show a weakened smoltification process and thereby is more heterogeneous than the anadromous stock might be revealed by physiological studies and an analysis of a representative subset of differential expressed genes from different functional groups (Table 2). Physiological studies of the same experimental fish, involving several parameters of smoltification, are in good agreement with the microarray data and indicate that similarity of smoltification between landlocked and anadromous salmon exists also at the functional level (Piironen et al. 2013). Thus, physiological studies involving a much larger number of fish individuals strongly support the gene expression data on the genetic similarity of the smoltification process in land-locked and anadromous salmon populations and suggest that pooling the samples is not concealing inter-individual differences in gene expression. Collectively, a microarray analysis and physiological studies provide compelling evidence for the hypothesis that some (but not all) landlocked salmon populations have retained similar smolt characteristics as anadromous salmon stocks and that smoltification in the landlocked salmon serves some important biological function.

Genetic basis of the parr-smolt transformation in the landlocked freshwater-resident salmon stock is qualitatively and quantitatively similar to that in the seawater migrating salmon. Extensive changes in gene expression and associated hypoosmotic adjustments of the gill constitute a futile energy-consuming sink for the freshwater salmon, but have been retained possibly due to their signaling function for migration between productively variable freshwater habitats. A comparison of gene expression between stunted and fast growing landlocked populations would probably provide further information about the biological importance of smoltification in freshwater salmon stocks. 


\section{Acknowledgements}

We thank Dr. Mikael Brosché (University of Helsinki, Finland) for guidance in microarray techniques. Jaakko Haverinen, Anita Kervinen and Riitta Pietarinen are appreciated for skilful technical assistance. The personnel of the Enonkoski hatchery are acknowledged for good care of the fish. Funding was provided by The Academy of Finland (grants 210400, 119583 and 127192) to Matti Vornanen and a grant from the joint research center for Environmental Risk Assessment of the Universities of Kuopio and Joensuu to Juha Lemmetyinen.

\section{References}

Alcorn, S. W., Murray, A. L. \& Pascho, R. J. 2002: Effects of rearing temperature on immune functions in sockeye salmon (Oncorhynchus nerka). - Fish and Shellfish Immunology 12: 303-334.

Ban, M., Ando, H. \& Urano, A. 2007: Effects of long-day on gill $\mathrm{Na}^{+}, \mathrm{K}^{+}$-ATPase gene expression and the development of seawater tolerance in sockeye salmon. - Aquaculture 273: 218-226.

Barbour, S. E. \& Garside, E. T. 1983: Some physiologic distinctions between freshwater and diadromous forms of the Atlantic salmon, Salmo salar L. - Canadian Journal of Zoology 61: 1165-1170.

Berg, O. K. 1985: The formation of non-anadromous populations of Atlantic salmon, Salmo salar L., in Europe. Journal of Fish Biology 27: 805-815.

Birt, T. P. \& Green, J. M. 1993: Acclimation to seawater of dwarf nonanadromous Atlantic salmon, Salmo salar. Canadian Journal of Zoology 71: 1912-1916.

Birt, T. P., Green, J. M. \& Davidson, W. S. 1991: Contrasts in development and smolting of genetically distinct sympatric anadromous and nonanadromous Atlantic salmon, Salmo salar. - Canadian Journal of Zoology 69: 2075-2084.

Bly, J. E. \& Clem, L. W. 1992: Temperature and teleost immune functions. - Fish and Shellfish Immunology 2: $159-171$.

Boeuf, G., Le Roux, A., Gaignon, J. L. \& Harache, Y. 1985: Gill $\left(\mathrm{Na}^{+}, \mathrm{K}^{+}\right)$-ATPase activity and smolting in Atlantic salmon (Salmo salar L.) in France. - Aquaculture 45: 73-81.

Brazma, A., Hingamp, P., Quackenbush, J., Sherlock, G., Spellman, P., Stoeckert, C., Aach, J., Ansorge, W., Ball, C. A., Causton, H. C., Gaasterland, T., Glenisson, P., Holstege, F. C. P., Kim, I. F., Markowitz, V., Matese, J. C., Parkinson, H., Robinson, A., Sarkans, U., SchulzeKremer, S., Stewart, J., Taylor, R., Vilo, J. \& Vingron, M. 2001: Minimum information about a microarray experiment (MIAME) - toward standards for microarray data. - Nature Genetics 29: 365-371.

Brosché, M., Vinocur, B., Alatalo, E. R., Lamminmäki, A., Teichmann, T., Ottow, E. A., Djilianov, D., Afif, D., Bogeat-Triboulot, M.-B., Altman, A., Polle, A., Dreyer, E., Rudd, S., Paulin, L., Auvinen, P. \& Kangasjärvi, J.
2005: Gene expression and metabolite profiling of Рориlus euphratica growing in the Negev desert. - Genome Biology 6: R101, doi:10.1186/gb-2005-6-12-r101.

Burton, M. P. \& Idler, D. R. 1984: Can Newfoundland landlocked salmon, Salmo salar L., adapt to sea water? Journal of Fish Biology 24: 59-64.

Bystriansky, J. S., Richards, J. G., Schulte, P. M. \& Ballantyne, J. S. 2006: Reciprocal expression of gill $\mathrm{Na}^{+}$/ $\mathrm{K}^{+}$-ATPase $\alpha$-subunit isoforms $\alpha 1 \mathrm{a}$ and $\alpha 1 \mathrm{~b}$ during seawater acclimation of three salmonid fishes that vary in their salinity tolerance. - Journal of Experimental Biology 209: 1848-1858.

Chernitsky, A. G. \& Loenko, A. A. 1983: The osmoregulatory system and possible ways of differentiation in ecological forms of Atlantic salmon, Salmo salar (Salmonidae). - Journal of Ichthyology 23: 84-94.

D'Cotta, H. C., Gallais, C., Saulier, B. \& Prunet, P. 1996: Comparison between parr and smolt Atlantic salmon (Salmo salar) $\alpha$ subunit gene expression of $\mathrm{Na}^{+} / \mathrm{K}^{+}$ ATPase in gill tissue. - Fish Physiology and Biochemistry 15: 29-39.

Dahl, K. 1928: The "blege" or dwarf-salmon. - Skrifter utgiven av det norske videnskapsakademi, Oslo.

Espelid, S., Løkken, G. B., Steiro, K. \& Bøgwald, J. 1996: Effects of cortisol and stress on the immune system in Atlantic Salmon (Salmo salar L.). - Fish and Shellfish Immunology 6: 95-110.

Evans, D. H., Piermarini, P. M. \& Choe, K. P. 2005: The multifunctional fish gill: dominant site of gas exchange, osmoregulation, acid-base regulation, and excretion of nitrogenous waste. - Physiological Reviews 85: 97-177.

Folmar, L. C. \& Dickhoff, W. W. 1980: The parr-smolt transformation (smoltification) and seawater adaptation in salmonids. - Aquaculture 21: 1-37.

Götz, S., García-Gómez, J. M., Terol, J., Williams, T. D., Nagaraj, S. H., Nueda, M. J., Robles, M., Talón, M., Dopazo, J. \& Conesa, A. 2008: High-throughput functional annotation and data mining with the Blast2GO suite. - Nucleic Acids Research 36: 3420-3435.

Hassinen, M., Paajanen, V., Haverinen, J., Eronen, H. \& Vornanen, M. 2007: Cloning and expression of cardiac $\mathrm{K}_{\mathrm{ir}} 2.1$ and $\mathrm{K}_{\mathrm{ir}} 2.2$ channels in thermally acclimated rainbow trout. - American Journal of Physiology 292: R2328-R2339.

Hoar, W. S. 1976: Smolt transformation: evolution, behavior and physiology. - Journal of the Fisheries Research Board of Canada 33: 1233-1252.

Hyndman, K. A. \& Evans, D. H. 2009: Effects of environmental salinity on gill endothelin receptor expression in the killifish, Fundulus heteroclitus. - Comparative Biochemistry and Physiology 152A: 58-65.

Kato, A., Nakamura, K., Kudo, H., Tran, Y. H., Yamamoto, Y., Doi, H. \& Hirose, S. 2009: Characterization of the column and autocellular junctions that define the vasculature of gill lamellae. - Journal of Histochemistry and Cytochemistry 55: 941-953.

Kiiskinen, P., Hyvärinen, H. \& Piironen, J. 2002: Smolting and seasonal variation in the smolt characteristics of one- and two-year-old Saimaa landlocked salmon under fish farm conditions. - Journal of Fish Biology 60: 
1015-1030.

Koch, H. J. A. E. 1983: Régulation minérale et hémoglobines chez quelques saumons (Salmo salar L.) dulçaquicoles. - Annales de la Société Royale Zoologique de Belgique 113 (suppl. 1): 259-270.

Komourdian, M. P., Saunders, R. L. \& Fenwick, J. C. 1976: Evidence for the role of growth hormone as a part of a "light-pituitary axis" in growth and smoltification of Atlantic salmon (Salmo salar). - Canadian Journal of Zoology 54: 544-551.

Kudo, H., Kato, A. \& Hirose, S. 2007: Fluorescence visualization of branchial collagen columns embraced by pillar cells. - Journal of Histochemistry and Cytochemistry 55: 57-62.

Langdon, J. S. 1985: Smoltification physiology in the culture of salmonids. - In: Muir, J. F. \& Roberts, R. J. (eds.), Recent advances in aquaculture, vol. 2: 79-118. Croom Helm, London.

Langdon, J. S. \& Thorpe, J. E. 1985: The ontogeny of smoltification: developmental patterns of gill $\mathrm{Na}^{+} / \mathrm{K}^{+}$-ATPase, $\mathrm{SDH}$, and chloride cells in juvenile Atlantic salmon, Salmo salar L. - Aquaculture 45: 83-95.

Madsen, S. S., Kiilerich, P. \& Tipsmark, C. K. 2009: Multiplicity of expression of $\mathrm{Na}^{+}, \mathrm{K}^{+}$-ATPase $\alpha$-subunit isoforms in the gill of Atlantic salmon (Salmo salar): cellular localisation and absolute quantification in response to salinity change. - Journal of Experimental Biology 212: 78-88.

McCormick, S. D. 1996: Effects of growth hormone and insulin-like growth factor I on salinity tolerance and gill $\mathrm{Na}^{+}, \mathrm{K}^{+}$-ATPase in Atlantic salmon (Salmo salar): interaction with cortisol. - General and Comparative Endocrinology 101: 3-11.

McCormick, S. D. \& Saunders, R. L. 1987: Preparatory physiological adaptations for marine life of salmonids: osmoregulation, growth, and metabolism. - American Fisheries Society Symposium 1: 211-229.

McCormick, S. D., Shrimpton, J. M., Moriyama, S. \& Björnsson, B. T. 2007: Differential hormonal responses of Atlantic salmon parr and smolt to increased daylength: a possible developmental basis for smolting. - Aquaculture 273: 337-344.

McDowall, R. M. 1988: Life history deviation and landlocking. - In: McDowall, R. M. (ed.), Diadromy in fishes: migrations between freshwater and marine environments: 171-185. Chapman \& Hall, London.

Mesa, M. G., Maule, A. G., Poe, T. P. \& Schreck, C. B. 1999: Influence of bacterial kidney disease on smoltification in salmonids: is it a case of double jeopardy? - Aquaculture 174: 25-41.

Mistry, A. C., Kato, A., Tran, Y. H., Honda, S., Tsukada, T., Takei, Y. \& Hirose, S. 2004: FHL5, a novel actinbinding protein, is highly expressed in eel gill pillar cells and responds to wall tension. - American Journal of Physiology 287: R1141-R1154.

Mommsen, T. P., Vijayan, M. M. \& Moon, T. W. 1999: Cortisol in teleosts: dynamics, mechanisms of action, and metabolic regulation. - Reviews in Fish Biology and Fisheries 9: 211-268.

Muona, M. \& Soivio, A. 1992: Changes in plasma lysozyme and blood leucocyte levels of hatchery-reared Atlantic salmon (Salmo salar L.) and sea trout (Salmo trutta L.) during parr-smolt transformation. - Aquaculture 106: $75-87$.

Nilsen, T. O., Ebbesson, L. O. E. \& Stefansson, S. O. 2003: Smolting in anadromous and landlocked strains of Atlantic salmon (Salmo salar). - Aquaculture 222: 71-82.

Nilsen, T. O., Ebbesson, L. O. E., Kiilerich, P., Björnsson, B. T., Madsen, S. S., McCormick, S. D. \& Stefansson, S. O. 2008: Endocrine systems in juvenile anadromous and landlocked Atlantic salmon (Salmo salar): seasonal development and seawater acclimation. - General and Comparative Endocrinology 155: 762-772.

Nilsen, T. O., Ebbesson, L. O. E., Madsen, S. S., McCormick, S. D., Andersson, E., Björnsson, B. T., Prunet, P. \& Stefansson, S. O. 2007: Differential expression of gill $\mathrm{Na}^{+}, \mathrm{K}^{+}$-ATPase $\alpha$ - and $\beta$-subunits, $\mathrm{Na}^{+}, \mathrm{K}^{+}, 2 \mathrm{Cl}^{-}$cotransporter and CFTR anion channel in juvenile anadromous and landlocked Atlantic salmon Salmo salar. - Journal of Experimental Biology 210: 2885-2896.

Parry, G. 1960: The development of salinity tolerance in the salmon, Salmo salar (L.) and some related species. Journal of Experimental Biology 37: 425-434.

Piironen, J., Kiiskinen, P., Huuskonen, H., Heikura-Ovaskainen, M. \& Vornanen, M. 2013: Comparison of smoltification in Atlantic salmon (Salmo salar L.) from anadromous and landlocked populations under common garden conditions. - Annales Zoologici Fennici 50: 1-15.

Rockett, J. C. \& Hellmann, G. M. 2004: Confirming microarray data - is it really necessary? - Genomics 83 : 541-549.

Rønneseth, A., Pettersen, E. F. \& Wergeland, H. I. 2005: Leucocytes of anadromous and landlocked strains of Atlantic salmon (Salmo salar L.) in the smolting period. - Fish and Shellfish Immunology 19: 229-239.

Saunders, R. L. \& Henderson, E. B. 1978: Changes in gill ATPase activity and smolt status of Atlantic salmon (Salmo salar). - Journal of the Fisheries Research Board of Canada 35: 1542-1546.

Schmitz, M. 1995: Seasonal changes in hypoosmoregulatory ability in landlocked and anadromous populations of Arctic charr, Salvelinus alpinus, and Atlantic salmon, Salmo salar. - Environmental Biology of Fishes 42: 401-412.

Seear, P. J., Carmichael, S. N., Talbot, R., Taggart, J. B., Bron, J. E. \& Sweeney, G. E. 2010: Differential gene expression during smoltification of Atlantic salmon (Salmo salar L.): a first large-scale microarray study. Marine Biotechnology 12: 126-140.

Seidelin, M., Madsen, S. S., Cutler, C. P. \& Cramb, G. 2001: Expression of gill vacuolar-type $\mathrm{H}^{+}$-ATPase $\beta$ subunit, and $\mathrm{Na}^{+}, \mathrm{K}^{+}$-ATPase $\alpha_{1}$ and $\beta_{1}$ subunit messenger RNAs in smolting Salmo salar. - Zoological Science 18: 315-324.

Smail, D. A., Bruno, D. W., Dear, G., McFarlane, L. A. \& Ross, K. 1992: Infectious pancreatic necrosis (IPN) virus Sp serotype in farmed Atlantic salmon, Salmo salar L., post-smolts associated with mortality and clinical disease. - Journal of Fish Diseases 15: 77-83.

Smyth, G. K. 2005: Limma: linear models for microarray 
data. - In: Gentleman, R., Carey, V., Dudoit, S., Irizarry, R. \& Huber, W. (eds.), Bioinformatics and computational biology. Solutions using $R$ and bioconductor: 397-420. Springer, New York.

Staurnes, M., Lysfjord, G. \& Berg, O. K. 1992: Parr-smolt transformation of a nonanadromous population of Atlantic salmon (Salmo salar) in Norway. - Canadian Journal of Zoology 70: 197-199.

Stensløkken, K. O., Sundin, L. \& Nilsson, G. E. 1999: Cardiovascular and gill microcirculatory effects of endothelin-1 in Atlantic cod: evidence for pillar cell contraction. - Journal of Experimental Biology 202: 1151-1157.

Sullivan, G. V., Fryer, J. N. \& Perry, S. F. 1996: Localization of mRNA for proton pump $\left(\mathrm{H}^{+}\right.$-ATPase $)$and $\mathrm{Cl}^{-} / \mathrm{HCO}_{3}^{-}$ exchanger in rainbow trout gill. - Canadian Journal of Zoology 74: 2095-2103.

Sutterlin, A. M. \& MacLean, D. 1984: Age at first maturity and the early expression of oocyte recruitment in two forms of Atlantic salmon (Salmo salar) and their hybrids. - Canadian Journal of Fisheries and Aquatic Sciences 41: 1139-1149.

Thorpe, J. E. 1994: An alternative view of smolting in salmonids. - Aquaculture 121: 105-113.

von Schalburg, K. R., Rise, M. L., Cooper, G. A., Brown, G. D., Gibbs, A. R., Nelson, C. C., Davidson, W. S. \& Koop, B. F. 2005: Fish and chips: various methodologies demonstrate utility of a 16,006-gene salmonid microarray. - BMC Genomics 6: 126.

Vornanen, M., Hassinen, M., Koskinen, H. \& Krasnov, A. 2005: Steady-state effects of temperature acclimation on the transcriptome of the rainbow trout heart. - American Journal of Physiology 289: R1177-R1184.

Vornanen, M., Lemmetyinen, J., Piironen, J. \& Kiiskinen, P. 2009: Gene expression of the gill epithelium in three salmon (Salmo salar) stocks migrating to freshwater, brackish water or full-strength seawater. - Comparative Biochemistry and Physiology 154(1), S3-S3, doi:10.1016/j.cbpa.2009.05.020. 